\title{
The wandering subspace property and Shimorin's condition of shift operator on the weighted Bergman spaces
}

\author{
Changhui Wu ${ }^{1} \cdot$ Zhijie Wang $^{2} \cdot$ Tao $\mathbf{Y u}^{3}$
}

Received: 21 May 2021 / Accepted: 21 September 2021 / Published online: 30 October 2021

(c) The Author(s) 2021

\begin{abstract}
In the present paper, we first study the wandering subspace property of the shift operator on the $I_{a}$ type zero based invariant subspaces of the weighted Bergman spaces $L_{a}^{2}\left(d A_{n}\right)(n=0,2)$ via the spectrum of some Toeplitz operators on the Hardy space $H^{2}$. Second, we give examples to show that Shimorin's condition for the shift operator fails on the $I_{a}$ type zero based invariant subspaces of the weighted Bergman spaces $L_{a}^{2}\left(d A_{\alpha}\right)(\alpha>0)$.
\end{abstract}

Keywords The weighted Bergman spaces - Quotient module $\cdot$ Beurling type theorem $\cdot$ Wandering subspace property

Mathematics Subject Classification 47A15 - 47A20

\section{Introduction}

Let $T$ be a bounded linear operator on a Hilbert space $H$. We say that $M$ is an invariant subspace for $T$ if $M$ is a closed subspace of $H$ and $T f \in M$ whenever $f \in M$. Let $\mathbb{D}$ be the open unit disk in the complex plane, and let $H(\mathbb{D})$ be the space consisting of all analytic functions on $\mathbb{D}$. The Hardy space $H^{2}$ is defined as:

Communicated by Dechao Zheng.

Zhijie Wang

wangzhijie628@zjxu.edu.cn

Changhui Wu

1083001701@qq.com

Tao Yu

tyu@dlut.edu.cn

1 School of Mathematical Sciences, Qufu Normal University, Jining, China

2 College of Data Science, Jiaxing University, Jiaxing, China

3 School of Mathematical Sciences, Dalian University of Technology, Dalian, China 


$$
H^{2}=\left\{f \in H(\mathbb{D}): f(z)=\sum_{n=0}^{\infty} a_{n} z^{n}, \sum_{n=0}^{\infty}\left|a_{n}\right|^{2}<\infty\right\},
$$

where $f(z)=\sum_{n=0}^{\infty} a_{n} z^{n}$ is the power series representation of $f$. Let $\mathbb{T}$ be the boundary of the unit disk $\mathbb{D}$. A function $\phi(z)$ in $H^{2}$ is called inner if $|\phi(z)|=1$ a.e. on $\mathbb{T}$. The famous Beurling theorem [2] says that every invariant subspace of the multiplication operator $T_{z}$ on the Hardy space $H^{2}$ other than $\{0\}$ has the form $\phi H^{2}$, where $\phi(z)$ is an inner function. To generalized the Beurling theorem, for a bounded linear operator $T$ on a Hilbert space $H$, let $M \ominus T M$ be the orthogonal complement of $T M$ in $M$, it is also called a wandering subspace of $T$ on $M$. We say that $T$ possesses the wandering subspace property on the invariant subspace $M$ of $T$ if:

$$
[M \ominus T M]=M
$$

where $[M \ominus T M]$ is the invariant subspace of $T$ generated by $M \ominus T M$. And we say that the Beurling type theorem for $T$ holds on $H$ if (1.1) holds for all invariant subspaces $M$ of $T$. The Beurling theorem implies that the Beurling type theorem holds for $T_{z}$ on $H^{2}$. The following is a basic problem in functional analysis:

Question 1 Describe the invariant subspaces of $T$ and find whether $T$ possesses the wandering subspace property on these invariant subspaces.

Let $d A$ be the normalized Lebesgue area measure on $\mathbb{D}$, and let:

$$
d A_{\alpha}(z)=(\alpha+1)\left(1-|z|^{2}\right)^{\alpha} d A(z)(\alpha>-1) .
$$

The space $L^{2}\left(\mathbb{D}, d A_{\alpha}\right)$ consists of complex valued functions $f$ on $\mathbb{D}$ such that:

$$
\|f\|=\left[\int_{\mathbb{D}}|f(z)|^{2} d A_{\alpha}(z)\right]^{\frac{1}{2}}<+\infty .
$$

It is well-known that $L^{2}\left(\mathbb{D}, d A_{\alpha}\right)$ is a Hilbert space with the above norm. For any $\alpha>-1$, we define:

$$
L_{a}^{2}\left(d A_{\alpha}\right)=H(\mathbb{D}) \cap L^{2}\left(\mathbb{D}, d A_{\alpha}\right),
$$

then $L_{a}^{2}\left(d A_{\alpha}\right)$ is a closed subspace of $L^{2}\left(\mathbb{D}, d A_{\alpha}\right)$. These spaces will be called the weighted Bergman spaces. Let $\mathcal{B}_{\alpha}$ denote the shift operator on $L_{a}^{2}\left(d A_{\alpha}\right)$ which maps every $f \in L_{a}^{2}\left(d A_{\alpha}\right)$ to $z f$. If $\alpha=0$, for convenience, the Bergman space $L_{a}^{2}\left(d A_{0}\right)$ and the Bergman shift $\mathcal{B}_{0}$ will be denoted by $L_{a}^{2}$ and $\mathcal{B}$, respectively.

In 1996, Aleman, Richter and Sundberg [1] proved that the Beurling type theorem holds for $\mathcal{B}$ on $L_{a}^{2}$. Later, different proofs of the Beurling theorem for $\mathcal{B}$ on $L_{a}^{2}$ were given in $[6,7,9]$. In [9], Shimorin proved the following theorem.

Theorem 1.1 (Shimorin's theorem) Let $T$ be a linear operator on a Hilbert space $H$ with the properties: 
(i) $\|T x+y\|^{2} \leq 2\left(\|x\|^{2}+\|T y\|^{2}\right)$ for allx, $y \in H$, and

(ii) $\cap_{n=1}^{\infty} T^{n} H=\{0\}$, then $T$ possesses the wandering subspace property on $H$.

Remark 1.2 The above condition (i) is equivalent to say that $T$ is bounded and bounded below on $H$ and satisfies the following equation:

$$
T T^{*}+\left(T^{*} T\right)^{-1} \leq 2 I .
$$

If $T$ satisfies the condition (ii) on $H$, then we say that $T$ is analytic on $H$.

Definition 1.3 We say that Shimorin's condition for $T$ holds on $H$ if $T$ satisfies the above conditions (i) and (ii) (see [12]).

Shimorin's theorem implies that $T$ possesses the wandering subspace property on $H$ if Shimorin's condition for $T$ holds on $H$. As an application of Shimorin's theorem, it is proved that for any $-1<\alpha \leq 0$ the Beurling type theorem holds for $\mathcal{B}_{\alpha}$ on $L_{a}^{2}\left(d A_{\alpha}\right)$, and then as a corollary, they gave a simpler proof of the Beurling type theorem on the Bergman space. One year later, Shimorin proved that for any $-1<\alpha \leq 1$ the Beurling-type theorem holds for $\mathcal{B}_{\alpha}$ on $L_{a}^{2}\left(d A_{\alpha}\right)$ (see [10]). The first step to solve Question 1 is to verify whether Shimorin's condition for the operator $T$ holds on the invariant subspaces. It is always difficult to verify directly according to Definition 1.3, for example, the reproducing kernel Hilbert spaces with the complicate kernel functions. If Shimorin's condition for the operator $T$ fails on some invariant subspaces, then we must try other ways to solve Question 1.

The famous example is the zero-based invariant subspaces of the weighted Bergman spaces $L_{a}^{2}\left(d A_{\alpha}\right)(\alpha>-1)$.

Definition 1.4 For any $n \geq 1, \alpha>-1$, now suppose the sequence $A=\left\{a_{1}, a_{2}, \ldots, a_{n}, \ldots\right\} \subset \mathbb{D}($ may be same $)$. Let:

$$
I_{A}=\left\{f \in L_{a}^{2}\left(d A_{\alpha}\right): f\left(a_{1}\right)=f\left(a_{2}\right)=\cdots=f\left(a_{n}\right)=\cdots=0\right\},
$$

then $I_{A}$ is an invariant subspace of $\mathcal{B}_{\alpha}$. The subspace $I_{A}$ is called a zero based invariant subspace of $L_{a}^{2}\left(d A_{\alpha}\right)$ (see [3]). When $A=\left\{a_{1}, a_{2}, \ldots, a_{n}\right\}$ is a finite set of points inside $\mathbb{D}$, for distinguishing the difference, we denote by:

$$
I_{a_{1}, a_{2}, \ldots, a_{n}}=I_{A} \text {. }
$$

In particular, let $A=\{a\}$, the kernel function for the $I_{a}$ type zero based invariant subspaces of $L_{a}^{2}\left(d A_{\alpha}\right)$ (see [3]) is:

$$
K_{a}^{\alpha}(z, w)=\frac{1}{(1-\bar{w} z)^{\alpha+2}}-\frac{\left(1-|a|^{2}\right)^{\alpha+2}}{(1-\bar{a} z)^{\alpha+2}(1-\bar{w} a)^{\alpha+2}} .
$$

In the present paper, we give the following theorem to show that Shimorin's condition for the shift operator fails on the $I_{a}$ type zero-based invariant subspaces of the weighted Bergman spaces $L_{a}^{2}\left(d A_{\alpha}\right)(\alpha>0)$. 
Theorem 1.5 If $1-|a|$ small enough, then Shimorin's condition fails for $\mathcal{B}_{\alpha}$ on the $I_{a}$ type zero based invariant subspaces of the weighted Bergman spaces $L_{a}^{2}\left(d A_{\alpha}\right)(\alpha>0)$; or equivalently,

$$
\left\langle\mathcal{B}_{\alpha} \mathcal{B}_{\alpha, I_{a}}^{*} f, f\right\rangle+\left\langle\left(\mathcal{B}_{\alpha, I_{a}}^{*} \mathcal{B}_{\alpha}\right)^{-1} f, f\right\rangle>2\|f\|^{2}
$$

holds for some $f \in I_{a}$ as $|a| \rightarrow 1^{-}$, where $\mathcal{B}_{\alpha, I_{a}}^{*}$ denotes the adjoint of the restriction $\left.\mathcal{B}_{\alpha}\right|_{I_{a}}$.

We study the zero based invariant subspaces of the weighted Bergman spaces is also due to the following facts:

Hedenmalm and Zhu [4] have showed that for any $\alpha>4$ there exists some $I_{a}$ type zero based invariant subspaces of $L_{a}^{2}\left(d A_{\alpha}\right)$ don't possess the wandering subspace property. So in this case, the Beurling type theorem for $\mathcal{B}_{\alpha}$ on $L_{a}^{2}\left(d A_{\alpha}\right)$ fails. In [9], Shimorin conjectured that the critical value for the Beurling type theorem on the weighted Bergman spaces $L_{a}^{2}\left(d A_{\alpha}\right)$ is $\alpha=1$. In 2004, Hedenmalm and Perdomo [3] essentially proved that the Beurling type theorem fails in $L_{a}^{2}\left(d A_{\alpha}\right)$ for $\alpha>c$, where $c \approx 1.04$. It is natural to discuss the following question:

Question 2 Does Shimorin's condition for $\mathcal{B}_{\alpha}$ hold on all $I_{a}$ type zero based invariant subspaces of $L_{a}^{2}\left(d A_{\alpha}\right)$ with $\alpha \in(1,+\infty)$ ?

Theorem 1.5 gives a negative answer to Question 2. Then we must try other ways to solve the following natural question:

Question 3 Does $\mathcal{B}_{\alpha}$ possess the wandering subspace property on the $I_{a}$ type zero based invariant subspaces of $L_{a}^{2}\left(d A_{\alpha}\right)$ with $\alpha \in(1,+\infty)$ ?

In [12], the authors gave a positive answer for the case of $\alpha=2$ of Question 3 and proved the case of $\alpha=2$ of Theorem 1.5. To solve Question 3, let $\mathbb{D}^{2}, \mathbb{T}^{2}$ be the bidisk and torus which are the Cartesian product of 2 copies of $\mathbb{D}$ and $\mathbb{T}$, respectively. The Hardy space $H^{2}\left(\mathbb{D}^{2}\right)$ over the bidisk $\mathbb{D}^{2}$ consists of holomorphic functions $f$ on $\mathbb{D}^{2}$ satisfying:

$$
\|f\|^{2}:=\sup _{0<r<1} \int_{\mathbb{T}^{2}}|f(r \zeta)|^{2} \mathrm{~d} \sigma(\zeta)<\infty,
$$

where $\sigma$ is the normalized Haar measure on $\mathbb{T}^{2}$. It is also well-known that $H^{2}\left(\mathbb{D}^{2}\right)$ is a Hilbert space. Let $M$ be a closed subspace of $H^{2}\left(\mathbb{D}^{2}\right)$, we say that $M$ is a submodule if $M$ is invariant under multiplication operators $T_{z}$ and $T_{w}$. In particular, for any subset $X \subset H^{2}\left(\mathbb{D}^{2}\right)$, let:

$$
[X]=\operatorname{clos}\left\{\operatorname{span}\left[A\left(\mathbb{D}^{2}\right) X\right]\right\},
$$

where $A\left(\mathbb{D}^{2}\right)$ is the bidisk algebra, that is the closure of polynomials in $z$ and $w$ under the norm of $H^{\infty}\left(\mathbb{D}^{2}\right)$. Then $[X]$ is a submodule, it is called the submodule generated 
by $X$. The orthonormal complement of a submodule is called a quotient module. Let $\left(S_{z}, S_{w}\right)$ be the two variable Jordan block which are the compression operators defined on a quotient module $N$, more precisely,

$$
S_{z} f=P_{N} z f, \quad S_{u} f=P_{N} w f, \quad \forall f \in N,
$$

where $P_{N}$ is the projection of $H^{2}\left(\mathbb{D}^{2}\right)$ onto $N$.

In particular, let $K_{1}=[z-w]$ be the submodule generated by $z-w$, and let $N_{1}=H^{2}\left(\mathbb{D}^{2}\right) \ominus K_{1}$ be the related quotient module. As we know, the quotient module $N_{1}$ plays a great role in many situations since the compression operator $S_{z}$ on $N_{1}$ is unitarily equivalent to the Bergman shift on $L_{a}^{2}$. In [11], by lifting the Bergman shift as $S_{z}$ on $N_{1}$, Sun and Zheng gave a proof of the Beurling type theorem for $\mathcal{B}$ on $L_{a}^{2}$. In [12], the authors considered the submodule $K_{0}=\left[(z-w)^{2}\right]$ and the related quotient module $N_{0}=H^{2}\left(\mathbb{D}^{2}\right) \ominus K_{0}$. Let $\mathcal{H}_{0}^{1}=[z-w] \ominus\left[(z-w)^{2}\right]$, then $N_{0}=N_{1} \oplus \mathcal{H}_{0}^{1}$, and the following lemma [12] holds:

Lemma 1.6 Define $S_{z}^{1}=\left.S_{z}\right|_{\mathcal{H}_{0}^{1}}$, then $S_{z}^{1}$ and $\mathcal{B}_{2}$ are unitarily equivalent.

The space $L^{\infty}(\mathbb{T})$ is the collection of all essentially bounded measurable functions on $\mathbb{T}$. For each $\phi\left(e^{i \theta}\right) \in L^{\infty}(\mathbb{T})$, the Toeplitz operator with symbol $\phi\left(e^{i \theta}\right)$ is the operator $T_{\phi}$ defined by:

$$
T_{\phi} f=P \phi f
$$

for each $f$ in $H^{2}$, where $P$ is the orthogonal projection of $L^{2}$ onto $H^{2}$. By some statements in [12], we get the following theorem:

Theorem 1.7 For any $0 \neq a \in \mathbb{D}$, then $I_{a} \neq\left[I_{a} \ominus \mathcal{B}_{2} I_{a}\right]$ holds in $L_{a}^{2}\left(d A_{2}\right)$ if only and if:

$$
\lambda_{a}=-\frac{1-\left(1-|a|^{2}\right)^{4}}{|a|^{2}\left(1-|a|^{2}\right)^{4}}
$$

is an eigenvalue of Toeplitz operator $T_{\phi}$ with an eigenvector

$$
f=\sum_{i=0}^{\infty} C_{3}^{i} z^{i}
$$

satisfying

$$
\sum_{i=0}^{\infty} \sum_{k=3}^{\infty}\left|C_{k}^{i}\right|^{2}<\infty
$$

where 


$$
\begin{aligned}
\phi\left(e^{i \theta}\right)= & \frac{1}{\left(1-|a|^{2}\right)^{2}}\left[\frac{1}{\left(1-a e^{-i \theta}\right)^{2}}-1\right] \\
& +\frac{1}{1-|a|^{2}}\left[\frac{1}{\left(1-a e^{-i \theta}\right)^{3}}-1\right] \\
& +\frac{1}{\left(1-|a|^{2}\right)^{3}}\left(\frac{1}{1-a e^{-i \theta}}-1\right),
\end{aligned}
$$

and

$$
C_{k+2}^{i}=(\sqrt{k})^{2} \sum_{j=0}^{k-1} \bar{a}^{k-1-j} C_{3}^{i+j}, \quad k \geq 2, i \geq 0 .
$$

The last part of the proof of Theorem 8 in [12] has in fact proved that $\lambda_{a} \notin \sigma\left(T_{\phi}\right)$. We believe that Theorem 1.7 is a general phenomenon in the case of all zero based invariant subspaces of $L_{a}^{2}\left(d A_{\alpha}\right)(\alpha>1)$. We also believe that $\mathcal{B}_{\alpha}$ does not possess the wandering subspace property on some zero-based invariant subspaces of $L_{a}^{2}\left(d A_{\alpha}\right)(\alpha>1)$ and it will substantiate Shimorin's conjecture. Our basic problem is to find and prove the general phenomenon, and then, we prove the wandering subspace property of $\mathcal{B}_{\alpha}$ on all zero based invariant subspaces of $L_{a}^{2}\left(d A_{\alpha}\right)(\alpha>1)$ via the spectrum of some Toeplitz operators on the Hardy space $H^{2}$. It is obvious that Question 3 is a special case of our basic problem. In the present paper, we prove the similar phenomenon in the case of Bergman space and reprove the wandering subspace property of the Bergman shift on the $I_{a}$ type zero-based invariant subspaces. The following is our main theorem:

Theorem 1.8 For any $0 \neq a \in \mathbb{D}$, then $I_{a} \neq\left[I_{a} \ominus \mathcal{B} I_{a}\right]$ holds in $L_{a}^{2}$ if only and if:

$$
\lambda_{a}=-\frac{1-\left(1-|a|^{2}\right)^{2}}{|a|^{2}\left(1-|a|^{2}\right)^{2}}
$$

is an eigenvalue of Toeplitz operator $T_{\phi}$ with an eigenvector:

$$
f=\sum_{i=0}^{\infty} C_{1}^{i} z^{i}
$$

satisfying:

$$
\sum_{i=0}^{\infty} \sum_{k=1}^{\infty}\left|C_{k}^{i}\right|^{2}<\infty
$$

where

$$
\phi\left(e^{i \theta}\right)=\frac{1}{1-|a|^{2}}\left(\frac{1}{1-a e^{-i \theta}}-1\right),
$$

and 


$$
C_{k}^{i}=(\sqrt{k})^{0} \sum_{j=0}^{k-1} \bar{a}^{k-1-j} C_{1}^{i+j}, \quad \forall k \geq 2, i \geq 0 .
$$

Through Theorems 1.7 and 1.8, we conjecture that the following proposition holds for the general case of the weighted Bergman spaces $L_{a}^{2}\left(d A_{n}\right)(n=0,1,2, \ldots)$

Conjecture 1 For any $0 \neq a \in \mathbb{D}$, then $I_{a} \neq\left[I_{a} \ominus \mathcal{B}_{n} I_{a}\right]$ holds in $L_{a}^{2}\left(d A_{n}\right)(n=0,1,2, \ldots)$ if only and if:

$$
\lambda_{a}=-\frac{1-\left(1-|a|^{2}\right)^{2+n}}{|a|^{2}\left(1-|a|^{2}\right)^{2+n}}
$$

is an eigenvalue of Toeplitz operator $T_{\phi}$ with an eigenvector

$$
f=\sum_{i=0}^{\infty} C_{n+1}^{i} z^{i}
$$

satisfying:

$$
\sum_{i=0}^{\infty} \sum_{k=n+1}^{\infty}\left|C_{k}^{i}\right|^{2}<\infty
$$

where

$$
\phi\left(e^{i \theta}\right)=\sum_{i=1}^{1+n} \frac{1}{\left(1-|a|^{2}\right)^{2+n-i}}\left(\frac{1}{\left(1-a e^{-i \theta}\right)^{i}}-1\right),
$$

and

$$
C_{k+n}^{i}=(\sqrt{k})^{n} \sum_{j=0}^{k-1} \bar{a}^{k-1-j} C_{n+1}^{i+j}, \quad k \geq 2, i \geq 0 .
$$

We believe that the proof of Conjecture 1 will make progress in solving Question 3, but we cannot prove it in this paper. This paper is arranged as follows. In Sect. 2, we prove Theorem 1.7. In Sect. 3, we give an equivalent condition that $S_{z}$ does not possess the wandering subspace property on any fixed invariant subspace of $S_{z}$ on $N_{1}$. In this part of the research process, we simply get the same characterization of $\mathcal{M}_{00}$ as in [11]. In Sect. 4, we give the proof of Theorem 1.8, and then as a corollary, we reprove that the Bergman shift possesses the wandering subspace property on all $I_{a}$ type zero based invariant subspaces. In Sect. 5, we prove Theorem 1.5.

In this paper, for a Hilbert space $H$ and a bounded linear operator $T$ on it, we denote by $\operatorname{Lat}(T)$ the lattice of invariant subspaces for $T$ on $H$. 


\section{The proof of theorem 1.7}

Let

$$
\begin{aligned}
& \tilde{\eta}_{k}=\sqrt{\frac{3}{k(k+1)(k+2)}} \sum_{i=0}^{k}(k-2 i) z^{i} w^{k-i}, \quad \forall k \geq 2, \\
& \tilde{\eta}_{1}=-\frac{1}{\sqrt{2}(z-w),}
\end{aligned}
$$

then $\left\{\tilde{\eta}_{1}, \tilde{\eta}_{2}, \ldots\right\}$ is an orthonormal basis of $\mathcal{H}_{0}^{1}$ (see [12]). In [12], the authors have proved the following proposition:

Proposition 2.1 (Corollary 2 in [12]) Let $\mathcal{M} \subset \mathcal{H}_{0}^{1}$ and $\mathcal{M} \in \operatorname{Lat}\left(S_{z}^{1}\right)$, then $\mathcal{M} \neq\left[\mathcal{M} \ominus S_{z}^{1} \mathcal{M}\right]_{\mathcal{H}_{0}^{1}}$ if and only if there exists a nonzero solution for the following equations of $C_{k}^{i}(k \geq 2, i \geq 0)$ :

(i) $\left\langle f_{i+1}^{0}, z\right\rangle+6 C_{2}^{i}=0(i \geq 0)$;

(ii) $-\sqrt{2}\left\langle f_{i+1}^{0}, \tilde{\eta}_{2}\right\rangle-4 C_{2}^{i+1}+\frac{10}{3} C_{3}^{i}=0(i \geq 0)$;

(iii) $\sqrt{\frac{3 k}{(k+1)(k+2)}}\left\langle f_{i+2}^{0}, \tilde{\eta}_{k}\right\rangle-\sqrt{\frac{3(k+3)}{(k+1)(k+2)}}\left\langle f_{i+1}^{0}, \tilde{\eta}_{k+1}\right\rangle+C_{k}^{i+2}-\frac{2(k+3)}{k+1} C_{k+1}^{i+1}+$

$$
\frac{(k+3)(k+4)}{(k+1)(k+2)} C_{k+2}^{i}=0(k \geq 2, i \geq 0) \text {; }
$$

(iv) $\sum_{i=0}^{\infty} \sum_{k=2}^{\infty}\left|C_{k}^{i}\right|^{2}<\infty$,

where

$$
\begin{aligned}
& f_{i}=\sum_{k=2}^{\infty} C_{k}^{i}\left(\sum_{t=1}^{k} \frac{6 t-2-4 k}{k(k-1)} z^{t} w^{k-t}+w^{k}\right), \\
& f_{i}^{0}=-S_{z}\left(\mathcal{B}_{\mathcal{M}}^{*} S_{z}\right)^{-1} P_{\mathcal{M}} T_{z}^{*} f_{i}
\end{aligned}
$$

for any $i \geq 0$, and $\tilde{\eta}_{1}, \tilde{\eta}_{2}, \ldots$ is an orthonormal basis of $\mathcal{H}_{0}^{1}$ as mentioned above.

In this section, we first point out that Proposition 2.1 can be written in the following simple form:

Theorem 2.2 Let $\mathcal{M} \subset \mathcal{H}_{0}^{1}$ and $\mathcal{M} \in \operatorname{Lat}\left(S_{z}^{1}\right)$, then $\mathcal{M} \neq\left[\mathcal{M} \ominus S_{z}^{1} \mathcal{M}\right]_{\mathcal{H}_{0}^{1}}$ if and only if there exists a nonzero solution for the following equations of $C_{k}^{i}(k \geq 3, i \geq 0)$ :

(a) $\left\langle f_{i+1}^{0}, \tilde{\eta}_{k}\right\rangle=-\frac{\sqrt{(k+1)(k+2)}}{\sqrt{3 k}} C_{k}^{i+1}+\frac{(k+3) \sqrt{k+2}}{\sqrt{3 k(k+1)}} C_{k+1}^{i}, \quad \forall i \geq 0, k \geq 2$;

(b) $\sum_{i=0}^{\infty} \sum_{k=3}^{\infty}\left|C_{k}^{i}\right|^{2}<\infty$,

where

Birkhäuser 


$$
\begin{gathered}
C_{2}^{i}=0, f_{i}=\sum_{k=2}^{\infty} C_{k}^{i}\left(\sum_{t=1}^{k} \frac{6 t-2-4 k}{k(k-1)} z^{t} w^{k-t}+w^{k}\right), \\
f_{i}^{0}=-S_{z}\left(\mathcal{B}_{\mathcal{M}}^{*} S_{z}\right)^{-1} P_{\mathcal{M}} T_{z}^{*} f_{i}
\end{gathered}
$$

for any $i \geq 0$, and $\tilde{\eta}_{1}, \tilde{\eta}_{2}, \ldots$ is an orthonormal basis of $\mathcal{H}_{0}^{1}$ as mentioned above.

Proof The condition (i) in Proposition 2.1 is equivalent to $C_{2}^{i}=0$ for any $i \geq 0$ (see [12]). If $C_{2}^{i}=0, i \geq 0$, then condition (ii) in Proposition 2.1 is

$$
\left\langle f_{i+1}^{0}, \tilde{\eta}_{2}\right\rangle=\frac{5 \sqrt{2}}{3} C_{3}^{i}, \quad \forall i \geq 0 .
$$

Then by (iii), we can calculate that

$$
\begin{aligned}
& \left\langle f_{i+1}^{0}, \tilde{\eta}_{3}\right\rangle=-\frac{2 \sqrt{5}}{3} C_{3}^{i+1}+\sqrt{5} C_{4}^{i} \\
& \left\langle f_{i+1}^{0}, \tilde{\eta}_{4}\right\rangle=-\frac{\sqrt{5}}{\sqrt{2}} C_{4}^{i+1}+\frac{7}{\sqrt{10}} C_{5}^{i} \\
& \left\langle f_{i+1}^{0}, \tilde{\eta}_{5}\right\rangle=-\frac{\sqrt{14}}{\sqrt{5}} C_{5}^{i+1}+\frac{8 \sqrt{7}}{3 \sqrt{10}} C_{6}^{i}, \ldots
\end{aligned}
$$

In general, we can prove that (ii) and (iii) are equivalent to:

$$
\begin{aligned}
\left\langle f_{i+1}^{0}, \tilde{\eta}_{k}\right\rangle= & -\frac{\sqrt{(k+1)(k+2)}}{\sqrt{3 k}} C_{k}^{i+1} \\
& +\frac{(k+3) \sqrt{k+2}}{\sqrt{3 k(k+1)}} C_{k+1}^{i}, \quad \forall i \geq 0, k \geq 2,
\end{aligned}
$$

where $C_{2}^{i}=0$ for all $i \geq 0$. In fact, if $k=2$, then (2.2) is becoming:

$$
\left\langle f_{i+1}^{0}, \tilde{\eta}_{2}\right\rangle=\frac{5 \sqrt{2}}{3} C_{3}^{i}, \quad \forall i \geq 0,
$$

that is (ii). If (ii) and (iii) hold, then (2.2) holds for $k=2$. We assume that (2.2) holds for any fixed $k \geq 2$, then by (iii),

$$
\begin{aligned}
& \sqrt{\frac{3 k}{(k+1)(k+2)}\left(-\frac{\sqrt{(k+1)(k+2)}}{\sqrt{3 k}} C_{k}^{i+2}+\frac{(k+3) \sqrt{k+2}}{\sqrt{3 k(k+1)}} C_{k+1}^{i+1}\right)} \\
& -\sqrt{\frac{3(k+3)}{(k+1)(k+2)}}\left\langle f_{i+1}^{0}, \tilde{\eta}_{k+1}\right\rangle+C_{k}^{i+2} \\
& -\frac{2(k+3)}{k+1} C_{k+1}^{i+1}+\frac{(k+3)(k+4)}{(k+1)(k+2)} C_{k+2}^{i}=0,
\end{aligned}
$$


that is:

$$
\begin{aligned}
\left\langle f_{i+1}^{0}, \tilde{\eta}_{k+1}\right\rangle= & -\frac{\sqrt{(k+2)(k+3)}}{\sqrt{3(k+1)}} C_{k+1}^{i+1} \\
& +\frac{(k+4) \sqrt{k+3}}{\sqrt{3(k+1)(k+2)}} C_{k+2}^{i}, \quad \forall i \geq 0,
\end{aligned}
$$

so (2.2) holds for $k+1$, by the induction, (2.2) holds. Conversely, if (2.2) holds, by the above calculations, then (ii) and (iii) hold.

Now, we give a proof of Theorem 1.7:

Lemma 2.3 (Theorem 7 in [12]) If $0 \neq a \in \mathbb{D}$, then $I_{a} \neq\left[I_{a} \ominus \mathcal{B}_{2} I_{a}\right]$ holds in $L_{a}^{2}\left(d A_{2}\right)$ if and only if there exists a nonzero solution for the following equations of $C_{3}^{i}(i \geq 0)$ :

(I)

$$
\begin{aligned}
& \sum_{n=1}^{\infty}(n+2)(n+3)\left(\bar{a}^{n-1} C_{3}^{i+1}+\bar{a}^{n-2} C_{3}^{i+2}+\cdots\right. \\
& \left.\quad+\bar{a} C_{3}^{i+n-1}+C_{3}^{i+n}\right) a^{n}=-2 \frac{1-\left(1-|a|^{2}\right)^{4}}{|a|^{2}\left(1-|a|^{2}\right)^{4}} C_{3}^{i}, \quad i \geq 0
\end{aligned}
$$

(II) $\sum_{i=0}^{\infty} \sum_{k=3}^{\infty}\left|C_{k}^{i}\right|^{2}<\infty$, where

$$
\begin{aligned}
C_{k+2}^{i}= & \frac{5(k+1)(k+2)}{6(k+4)}\left(\bar{a}^{k-1} C_{3}^{i}+\bar{a}^{k-2} C_{3}^{i+1}\right. \\
& \left.+\bar{a}^{k-3} C_{3}^{i+2}+\cdots+\bar{a} C_{3}^{i+k-2}+C_{3}^{i+k-1}\right), \quad k \geq 2, i \geq 0 .
\end{aligned}
$$

Proof of Theorem 1.7 By the proof of Theorem 8 in [12], we get that (2.5) has a nonzero solution $\left\{C_{3}^{i}\right\}_{i \geq 0}$ satisfying:

$$
\sum_{i=0}^{\infty}\left|C_{3}^{i}\right|^{2}<+\infty,
$$

which is equivalent to

$$
\lambda_{a}=-\frac{1-\left(1-|a|^{2}\right)^{4}}{|a|^{2}\left(1-|a|^{2}\right)^{4}}
$$

being an eigenvalue of Toeplitz operator $T_{\phi}\left(f=\sum_{i=0}^{\infty} C_{3}^{i} z^{i}\right.$ is an eigenvector), where 


$$
\begin{aligned}
\phi\left(e^{i \theta}\right)= & \frac{1}{\left(1-|a|^{2}\right)^{2}}\left[\frac{1}{\left(1-a e^{-i \theta}\right)^{2}}-1\right] \\
& +\frac{1}{1-|a|^{2}}\left[\frac{1}{\left(1-a e^{-i \theta}\right)^{3}}-1\right] \\
& +\frac{1}{\left(1-|a|^{2}\right)^{3}}\left(\frac{1}{1-a e^{-i \theta}}-1\right) .
\end{aligned}
$$

By Lemma 2.3, we get Theorem 1.7.

\section{Beurling type theorem for $S_{z}$ on $N_{1}$}

In this section, we use the techniques in [12].

Proposition $3.1 S_{z}$ is left invertible and analytic on $N_{1}$ [5].

Proof Note that an orthonormal basis of $N_{1}$ is:

$$
\left\{\tilde{e}_{0}, \tilde{e}_{1}, \ldots, \tilde{e}_{k}, \ldots\right\}
$$

where

$$
\tilde{e}_{k}=\frac{\sum_{i=0}^{k} z^{i} w^{k-i}}{\sqrt{k+1}}, \quad k \geq 0 .
$$

Then, we get the matrix $A$ of $S_{z}$ under the above basis:

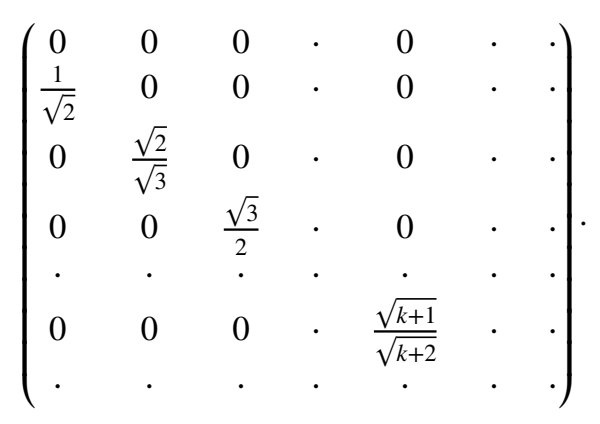

And one calculates $A^{*}$ and $A^{*} A$, respectively: 


$$
\begin{aligned}
& \left(\begin{array}{cccccccc}
0 & \frac{1}{\sqrt{2}} & 0 & 0 & . & 0 & \cdot & \cdot \\
0 & 0 & \frac{\sqrt{2}}{\sqrt{3}} & 0 & . & 0 & \cdot & . \\
0 & 0 & 0 & \frac{\sqrt{3}}{2} & \cdot & 0 & \cdot & \cdot \\
\cdot & \cdot & \cdot & \cdot & \cdot & \cdot & \cdot & \cdot \\
0 & 0 & 0 & 0 & \cdot & \frac{\sqrt{k}}{\sqrt{k+1}} & \cdot & \cdot \\
\cdot & \cdot & . & . & . & \cdot & . & .
\end{array}\right)
\end{aligned}
$$

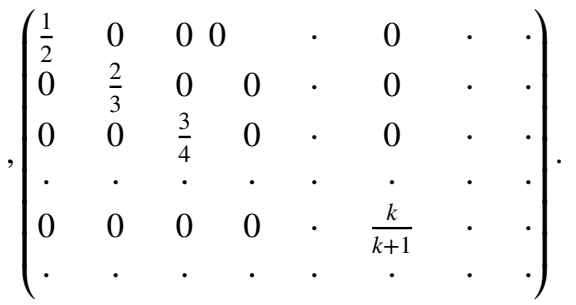

Since $A^{*} A$ is invertible(i.e. $S_{z}^{*} S_{z}$ is invertible), and therefore, $S_{z}$ is left invertible.

Since, for any $f \in N_{1}$, there exists a sequence $\left\{a_{n}\right\}_{n=0}^{\infty}$ such that:

$$
f=a_{0} \tilde{e}_{0}+a_{1} \tilde{e}_{1}+\ldots
$$

Then the coordinate vector of $S_{z} f$ is

$$
A\left(a_{0}, a_{1}, \ldots, a_{n}, \ldots\right)^{\prime}=\left(0, \frac{1}{\sqrt{2}} a_{0}, \frac{\sqrt{2}}{\sqrt{3}} a_{1}, \ldots, \frac{\sqrt{k+1}}{\sqrt{k+2}} a_{k}, \ldots\right)^{\prime} .
$$

Now if $g \in \cap_{n=1}^{\infty} S_{z}^{n} N_{1}$, there exists $f_{n} \in N_{1}$ such that:

$$
g=S_{z}^{n} f_{n}, \quad \forall n \geq 1 .
$$

For any fixed $n \geq 1$, comparing the coordinate vectors of $g$ and $S_{z}^{n} f_{n}$, we get that the fist $n$ numbers of coordinate vector of $g$ are zero, thus $g=0$. Hence $\cap_{n=1}^{\infty} S_{z}^{n} N_{1}=\{0\}$, i.e., $S_{z}$ is analytic.

For any $\mathcal{M} \in \operatorname{Lat}\left(S_{z}\right)$, let $\widetilde{\mathcal{M}}$ be the direct sum $\widetilde{\mathcal{M}}=\mathcal{M} \oplus[z-w]$. It is easy to verify that $\widetilde{\mathcal{M}}$ is an invariant subspace for $T_{z}$ in $H^{2}\left(\mathbb{D}^{2}\right)$. In Theorem 6.8 of [8], Richter showed that the mapping $\eta: \mathcal{M} \rightarrow \widetilde{\mathcal{M}}$ is one-to-one correspondence between invariant subspaces of $S_{z}$ and invariant subspaces of $T_{z}$ containing $[z-w]$. Let $\mathcal{L}_{\widetilde{\mathcal{M}}}$ be the wandering space of $T_{z}$ on $\widetilde{\mathcal{M}}$, we easily get the following theorem:

Theorem 3.2 Let $\mathcal{M} \in \operatorname{Lat}\left(S_{z}\right)$, we have the following decomposition:

$$
\widetilde{\mathcal{M}}=\sum_{n=0}^{\infty} \oplus z^{n} \mathcal{L}_{\widetilde{\mathcal{M}}}
$$


Proof Since for any function $f \in \bigcap_{n=0}^{\infty} T_{z}^{n} \widetilde{\mathcal{M}}, f$ and its derivatives vanish at $z=0$, we have $\bigcap_{n=0}^{\infty} T_{z}^{n} \widetilde{\mathcal{M}}=\{0\}$. By the Wold decomposition theorem, we have:

$$
\widetilde{\mathcal{M}}=\sum_{n=0}^{\infty} \oplus z^{n} \mathcal{L}_{\widetilde{\mathcal{M}}}
$$

Now, let $\mathcal{M}_{0}$ be the wandering space of $S_{z}$ on $\mathcal{M}$. Clearly, $\mathcal{M}_{0} \subset \mathcal{L}_{\widetilde{\mathcal{M}}}$. Let $\mathcal{M}_{00}$ be the orthogonal complement of $\mathcal{M}_{0}$ in $\mathcal{L}_{\widetilde{\mathcal{M}}}$. In [11], Sun and Zheng gave a description of $\mathcal{M}_{00}$. In the following discussion, we give other equivalent forms of $\mathcal{M}_{00}$.

\section{Lemma 3.3 [5] Let}

$$
X_{n}=\frac{1}{\sqrt{n+2}}\left(\frac{\sum_{i=0}^{n} z^{i+1} w^{n-i}}{\sqrt{n+1}}-\sqrt{n+1} w^{n+1}\right), \quad \forall n \geq 0 .
$$

Then $\left\{X_{n}\right\}_{n \geq 0}$ is an orthonormal basis of $K_{1} \ominus z K_{1}$.

Lemma 3.4 Let $\mathcal{M} \in \operatorname{Lat}\left(S_{z}\right)$, and let $P_{\mathcal{M}}$ be the projection of $N_{1}$ onto $\mathcal{M}$. Define $\mathcal{B}_{\mathcal{M}}^{*}$ to be the compression operator of $T_{z}^{*}$ on $\mathcal{M}$, i.e.,

$$
\mathcal{B}_{\mathcal{M}}^{*} q=P_{\mathcal{M}} T_{z}^{*} q, \quad \forall q \in \mathcal{M}
$$

Then $\mathcal{B}_{\mathcal{M}}^{*} S_{z}$ is invertible on $\mathcal{M}$.

Proof By Proposition 3.1, there exists a constant $C>0$, such that:

$$
\left\|S_{z} f\right\| \geq C\|f\|, \quad \forall f \in \mathcal{M}
$$

The Cauchy-Schwarz inequality gives:

$$
\left\|\mathcal{B}_{\mathcal{M}}^{*} S_{z} f\right\|\|f\| \geq\left|\left\langle\mathcal{B}_{\mathcal{M}}^{*} S_{z} f, f\right\rangle\right|=\left\|S_{z} f\right\|^{2} \geq C^{2}\|f\|^{2}
$$

Thus, we have:

$$
\left\|\mathcal{B}_{\mathcal{M}}^{*} S_{z} f\right\| \geq C^{2}\|f\|, \quad \forall f \in \mathcal{M}
$$

this implies that $\mathcal{B}_{\mathcal{M}}^{*} S_{z}$ is bounded below on $\mathcal{M}$. On the other hand, note that $\mathcal{B}_{\mathcal{M}}^{*} S_{z}=\left(\left.S_{z}\right|_{\mathcal{M}}\right)^{*} S_{z}$ is an adjoint operator on $\mathcal{M}$, then $\mathcal{B}_{\mathcal{M}}^{*} S_{z}$ is invertible on $\mathcal{M}$.

Theorem 3.5 Let $\mathcal{M} \in \operatorname{Lat}\left(S_{z}\right)$, and let $P_{\mathcal{M}}$ be the projection of $N_{1}$ onto $\mathcal{M}$. Then,

(i) $\mathcal{M}_{00}=\left\{f_{1}+f_{2}: f_{1} \in S_{z} \mathcal{M}, f_{2} \in K_{1} \ominus z K_{1}\right.$, and $\left.\mathcal{B}_{\mathcal{M}}^{*} f_{1}+P_{\mathcal{M}} T_{z}^{*} f_{2}=0\right\}$;

(ii) $\mathcal{M}_{00}=\left\{-S_{z}\left(\mathcal{B}_{\mathcal{M}}^{*} S_{z}\right)^{-1} P_{\mathcal{M}} T_{z}^{*} f+f, f \in K_{1} \ominus z K_{1}\right\}$; 
(iii) $\mathcal{M}_{00}=\left\{-h_{g}+z P_{N_{1}} g-w g(w): g \in H^{2}(w), h_{g}=S_{z}\left(\mathcal{B}_{\mathcal{M}}^{*} S_{z}\right)^{-1} P_{\mathcal{M}} g\right\}$.

Proof Prove (i). For any $f \in \widetilde{\mathcal{M}}$, there exists $f_{1} \in \mathcal{M}, f_{2} \in K_{1}$, such that $f=f_{1}+f_{2}$. On the one hand, we have the following equivalent relation:

$$
\begin{aligned}
f & =f_{1}+f_{2} \in \mathcal{L}_{\widetilde{\mathcal{M}}} ; \\
& \Leftrightarrow\left\langle f_{1}+f_{2}, T_{z}\left(\mathcal{M} \oplus K_{1}\right)\right\rangle=0 ; \\
& \Leftrightarrow\left\langle f_{1}+f_{2}, T_{z}\left(g_{1}+g_{2}\right)\right\rangle=0, \text { for any } g_{1} \in \mathcal{M}, g_{2} \in K_{1} ; \\
& \Leftrightarrow\left\langle f_{1}, T_{z} g_{1}\right\rangle+\left\langle f_{2}, T_{z} g_{1}\right\rangle+\left\langle f_{2}, T_{z} g_{2}\right\rangle=0 ; \\
& \Leftrightarrow\left\langle\mathcal{B}_{\mathcal{M}}^{*} f_{1}, g_{1}\right\rangle+\left\langle P_{\mathcal{M}} T_{z}^{*} f_{2}, g_{1}\right\rangle+\left\langle f_{2}, T_{z} g_{2}\right\rangle=0 ; \\
& \Leftrightarrow\left\langle\mathcal{B}_{\mathcal{M}}^{*} f_{1}+P_{\mathcal{M}} T_{z}^{*} f_{2}, g_{1}\right\rangle+\left\langle f_{2}, T_{z} g_{2}\right\rangle=0 ; \\
& \Leftrightarrow\left\langle\mathcal{B}_{\mathcal{M}}^{*} f_{1}+P_{\mathcal{M}} T_{z}^{*} f_{2}, g_{1}\right\rangle+\left\langle P_{K_{1}} T_{z}^{*} f_{2}, g_{2}\right\rangle ; \\
& \Leftrightarrow \mathcal{B}_{\mathcal{M}}^{*} f_{1}+P_{\mathcal{M}} T_{z}^{*} f_{2}=P_{K_{1}} T_{z}^{*} f_{2}=0 ; \\
& \Leftrightarrow T_{z}^{*} f_{2} \in N_{1}, \mathcal{B}_{\mathcal{M}}^{*} f_{1}+P_{\mathcal{M}} T_{z}^{*} f_{2}=0 .
\end{aligned}
$$

On the other hand, if $f=f_{1}+f_{2} \in \mathcal{L}_{\widetilde{\mathcal{M}}}$, we also have the following equivalent relations:

$$
f=f_{1}+f_{2} \in \mathcal{M}_{00} \Leftrightarrow f_{1}+f_{2} \perp \mathcal{M}_{0} \Leftrightarrow f_{1} \perp \mathcal{M}_{0} \Leftrightarrow f_{1} \in S_{z} \mathcal{M} .
$$

Thus we have

$$
\begin{aligned}
f & =f_{1}+f_{2} \in \mathcal{M}_{00} \\
& \Leftrightarrow f_{1} \in S_{z} \mathcal{M}, T_{z}^{*} f_{2} \in N_{1}, \mathcal{B}_{\mathcal{M}}^{*} f_{1}+P_{\mathcal{M}} T_{z}^{*} f_{2}=0 ; \\
& \Leftrightarrow f_{1} \in S_{z} \mathcal{M}, f_{2} \in K_{1} \ominus z K_{1}, \mathcal{B}_{\mathcal{M}}^{*} f_{1}+P_{\mathcal{M}} T_{z}^{*} f_{2}=0 .
\end{aligned}
$$

Hence:

$$
\begin{aligned}
\mathcal{M}_{00}= & \left\{f_{1}+f_{2}: f_{1} \in S_{z} \mathcal{M}, f_{2} \in K_{1} \ominus z K_{1},\right. \\
& \text { and } \left.\mathcal{B}_{\mathcal{M}}^{*} f_{1}+P_{\mathcal{M}} T_{z}^{*} f_{2}=0\right\} .
\end{aligned}
$$

Prove (ii). For any $g$ in $\mathcal{M}_{00}$, by (3.3), there exists $g_{1} \in S_{z} \mathcal{M}, g_{2} \in K_{1} \ominus z K_{1}$ such that $g=g_{1}+g_{2}$ and $\mathcal{B}_{\mathcal{M}}^{*} g_{1}+P_{\mathcal{M}} T_{z}^{*} g_{2}=0$. Since, there exists $h \in \mathcal{M}$ such that $g_{1}=S_{z} h$, then $\mathcal{B}_{\mathcal{M}}^{*} g_{1}=\mathcal{B}_{\mathcal{M}}^{*} S_{z} h=-P_{M} T_{z}^{*} g_{2}$. By Lemma 3.4, we have:

$$
h=-\left(\mathcal{B}_{\mathcal{M}}^{*} S_{z}\right)^{-1} P_{\mathcal{M}} T_{z}^{*} g_{2}, g_{1}=S_{z} h=-S_{z}\left(\mathcal{B}_{\mathcal{M}}^{*} S_{z}\right)^{-1} P_{\mathcal{M}} T_{z}^{*} g_{2},
$$

then,

$$
g=-S_{z}\left(\mathcal{B}_{\mathcal{M}}^{*} S_{z}\right)^{-1} P_{\mathcal{M}} T_{z}^{*} g_{2}+g_{2}, g_{2} \in K_{1} \ominus z K_{1} .
$$

This implies that: 


$$
\mathcal{M}_{00} \subset\left\{-S_{z}\left(\mathcal{B}_{\mathcal{M}}^{*} S_{z}\right)^{-1} P_{\mathcal{M}} T_{z}^{*} f+f, f \in K_{1} \ominus z K_{1}\right\}
$$

Conversely, by (3.3) again,

$$
\left\{-S_{z}\left(\mathcal{B}_{\mathcal{M}}^{*} S_{z}\right)^{-1} P_{\mathcal{M}} T_{z}^{*} f+f, f \in K_{1} \ominus z K_{1}\right\} \subset \mathcal{M}_{00},
$$

then we get:

$$
\mathcal{M}_{00}=\left\{-S_{z}\left(\mathcal{B}_{\mathcal{M}}^{*} S_{z}\right)^{-1} P_{\mathcal{M}} T_{z}^{*} f+f, f \in K_{1} \ominus z K_{1}\right\} .
$$

Prove (iii). For any $f$ in $K_{1} \ominus z K_{1}$, by Lemma 3.3,

$$
f=\sum_{n=0}^{\infty} c_{n} X_{n}=\sum_{n=0}^{\infty} \frac{c_{n}}{\sqrt{n+2}}\left(\frac{\sum_{i=0}^{n} z^{i+1} w^{n-i}}{\sqrt{n+1}}-\sqrt{n+1} w^{n+1}\right),
$$

where $\left\{c_{n}\right\}_{n \geq 0} \subset \mathbb{C}$ and satisfies that $\sum_{n=0}^{\infty}\left|c_{n}\right|^{2}<\infty$. Then,

$$
T_{z}^{*} f=\sum_{n=0}^{\infty} \frac{c_{n}}{\sqrt{n+2}} \frac{\sum_{i=0}^{n} z^{i} w^{n-i}}{\sqrt{n+1}}=\sum_{n=0}^{\infty} \frac{c_{n}}{\sqrt{n+2}} \tilde{e}_{n} .
$$

Let:

$$
g(w)=\left(T_{z}^{*} f\right)(w, w)=\sum_{n=0}^{\infty} \frac{\sqrt{n+1}}{\sqrt{n+2}} c_{n} w^{n}, \quad \forall w \in \mathbb{D},
$$

since, $\sum_{n=0}^{\infty}\left|\frac{\sqrt{n+1}}{\sqrt{n+2}} c_{n}\right|^{2} \leq \sum_{n=0}^{\infty}\left|c_{n}\right|^{2}<\infty$, so $g \in H^{2}(w)$. Since

$$
P_{N_{1}} g=\sum_{k=0}^{\infty}\left\langle P_{N_{1}} g, \tilde{e}_{k}\right\rangle \tilde{e}_{k}=\sum_{k=0}^{\infty}\left\langle g, \tilde{e}_{k}\right\rangle \tilde{e}_{k}=\sum_{k=0}^{\infty} \frac{c_{k}}{\sqrt{k+2}} \tilde{e}_{k},
$$

then,

$$
\begin{aligned}
z P_{N_{1}} g-w g(w)= & \sum_{k=0}^{\infty} \frac{c_{k}}{\sqrt{k+2}}\left(\frac{\sum_{i=0}^{k} z^{i+1} w^{k-i}}{\sqrt{k+1}}\right. \\
& \left.-\sqrt{k+1} w^{k+1}\right)=f .
\end{aligned}
$$

Thus:

$$
P_{\mathcal{M}} T_{z}^{*} f=P_{\mathcal{M}} T_{z}^{*}\left(z P_{N_{1}} g-w g(w)\right)=P_{\mathcal{M}} g .
$$

The above implies that: 


$$
\begin{gathered}
\mathcal{M}_{00} \subset\left\{-h_{g}+z P_{N_{1}} g-w g(w): g \in H^{2}(w),\right. \\
\left.h_{g}=S_{z}\left(\mathcal{B}_{\mathcal{M}}^{*} S_{z}\right)^{-1} P_{\mathcal{M}} g\right\} .
\end{gathered}
$$

Conversely, for any $g$ in $H^{2}(w)$, let $g(w)=\sum_{n=0}^{\infty} d_{n} w^{n}$, where $\sum_{n=0}^{\infty}\left|d_{n}\right|^{2}<\infty$. Let $c_{n}=\frac{\sqrt{n+2}}{\sqrt{n+1}} d_{n}$, and let $f=\sum_{n=0}^{\infty} c_{n} X_{n}$. Since,

$$
\sum_{n=0}^{\infty}\left|c_{n}\right|^{2} \leq 2 \sum_{n=0}^{\infty}\left|d_{n}\right|^{2}<\infty
$$

then $f \in K_{1} \ominus z K_{1}$. By above calculations, we have $f=z P_{N_{1}} g-w g(w)$ and $P_{\mathcal{M}} T_{z}^{*} f=P_{\mathcal{M}} g$. Hence,

$$
\begin{gathered}
\left\{-h_{g}+z P_{N_{1}} g-w g(w): g \in H^{2}(w),\right. \\
\left.h_{g}=S_{z}\left(\mathcal{B}_{\mathcal{M}}^{*} S_{z}\right)^{-1} P_{\mathcal{M}} g\right\} \subset \mathcal{M}_{00},
\end{gathered}
$$

this completes the proof.

Corollary 3.6 For any $\mathcal{M} \in \operatorname{Lat}\left(S_{z}\right)$, let $\mathcal{N}$ be the orthogonal complement of $\left[\mathcal{M}_{0}\right]$ in M. Then

$$
\mathcal{N} \subset\left\{\sum_{n=0}^{\infty} z^{n} u_{n}: u_{n}=-S_{z}\left(\mathcal{B}_{\mathcal{M}}^{*} S_{z}\right)^{-1} P_{\mathcal{M}} T_{z}^{*} f_{n}+f_{n} \in \mathcal{M}_{00}\right\} .
$$

Proof Let $q \in \mathcal{N}$, by Theorems 3.2 and 3.5, we have:

$$
q=\sum_{k=0}^{\infty} z^{k} \tilde{m}_{k}+\sum_{n=0}^{\infty} z^{n} u_{n}
$$

where $\tilde{m}_{k} \in \mathcal{M}_{0}$ and $u_{n}=-S_{z}\left(\mathcal{B}_{\mathcal{M}}^{*} S_{z}\right)^{-1} P_{\mathcal{M}} T_{z}^{*} f_{n}+f_{n} \in \mathcal{M}_{00}$.

Since, $q \in \mathcal{N}$ and $q \perp \vee_{n \geq 0} S_{z}^{n} \mathcal{M}_{0}$, taking inner product of $q$ with $S_{z}^{k} \tilde{m}_{k}$ gives

$$
\begin{aligned}
0 & =\left\langle q, S_{z}^{k} \tilde{m}_{k}\right\rangle=\left\langle q, P_{N_{1}} z^{k} \tilde{m}_{k}\right\rangle=\left\langle q, z^{k} \tilde{m}_{k}\right\rangle \\
& =\left\langle z^{k} \tilde{m}_{k}, z^{k} \tilde{m}_{k}\right\rangle+\left\langle z^{k} u_{k}, z^{k} \tilde{m}_{k}\right\rangle \\
& =\left\|\tilde{m}_{k}\right\|^{2}+\left\langle u_{k}, \tilde{m}_{k}\right\rangle=\left\|\tilde{m}_{k}\right\|^{2} .
\end{aligned}
$$

This implies that $\tilde{m}_{k}=0$ for all $k \geq 0$. Thus each function $q$ in $\mathcal{N}$ has the following form:

$$
q=\sum_{n=0}^{\infty} z^{n} u_{n}
$$


Let: $\tilde{\mathcal{N}}=\left\{\sum_{n=0}^{\infty} z^{n} u_{n}: u_{n} \in \mathcal{M}_{00}\right\}$, by Corollary 3.6, we have $\mathcal{N} \subset \tilde{\mathcal{N}}$. Let $u_{n}=f_{n}^{0}+f_{n}$, where $f_{n}^{0}=-S_{z}\left(\mathcal{B}_{\mathcal{M}}^{*} S_{z}\right)^{-1} P_{\mathcal{M}} T_{z}^{*} f_{n}$. For convenience, denote by $h_{n}=-\left(\mathcal{B}_{\mathcal{M}}^{*} S_{z}\right)^{-1} P_{\mathcal{M}} T_{z}^{*} f_{n}$, then $f_{n}^{0}=S_{z} h_{n}$. Let:

$$
\begin{aligned}
f_{n} & =\sum_{k=0}^{\infty} C_{k}^{n} X_{k} \\
& =\sum_{k=0}^{\infty} \frac{C_{k}^{n}}{\sqrt{k+2}}\left(\frac{\sum_{i=0}^{k} z^{i+1} w^{k-i}}{\sqrt{k+1}}-\sqrt{k+1} w^{k+1}\right), \quad \forall n \geq 0,
\end{aligned}
$$

where $\sum_{k=0}^{\infty}\left|C_{k}^{n}\right|^{2}<\infty$.

Lemma 3.7 Let: $\tilde{\mathcal{N}}=\left\{\sum_{n=0}^{\infty} z^{n} u_{n}: u_{n} \in \mathcal{M}_{00}\right\}$. For any $f \in \tilde{\mathcal{N}}$, we have the decomposition:

$$
\begin{aligned}
& f=\sum_{n=0}^{\infty} z^{n} u_{n}, u_{n}=f_{n}^{0}+f_{n}, \\
& f_{n}=\sum_{k=0}^{\infty} \frac{C_{k}^{n}}{\sqrt{k+2}}\left(\frac{\sum_{i=0}^{k} z^{i+1} w^{k-i}}{\sqrt{k+1}}-\sqrt{k+1} w^{k+1}\right), \quad \forall n \geq 0 .
\end{aligned}
$$

Then, $f \in \mathcal{N}$ if and only if the following conditions are satisfying:

(i) $C_{0}^{i}=0(i \geq 0)$;

(ii) $\left\langle f_{i+1}^{0}, \tilde{e}_{k}\right\rangle-\sqrt{k} C_{k-1}^{i+1}+\sqrt{k+2} C_{k}^{i}=0, \quad \forall i \geq 0, k \geq 1$.

Proof Step 1. For any $f$ in $\tilde{\mathcal{N}}$, let $f=\sum_{n=0}^{\infty} z^{n} u_{n}$, where $u_{n} \in \mathcal{M}_{00}$. On the one hand, by Theorem 3.2, we have $f \in \mathcal{M}$. Thus $f \in \mathcal{M} \Leftrightarrow f \perp K_{1}$. On the other hand, assume $f \in \mathcal{M}$. Since for any $k \geq 0, \tilde{m}_{k} \in \mathcal{M}_{0}$, we have:

$$
\begin{aligned}
\left\langle\sum_{n=0}^{\infty} z^{n} u_{n}, S_{z}^{k} \tilde{m}_{k}\right\rangle & =\left\langle\sum_{n=0}^{\infty} z^{n} u_{n}, P_{N_{1}} z^{k} \tilde{m}_{k}\right\rangle \\
& =\left\langle\sum_{n=0}^{\infty} z^{n} u_{n}, z^{k} \tilde{m}_{k}\right\rangle \\
& =\left\langle z^{k} u_{k}, z^{k} \tilde{m}_{k}\right\rangle \\
& =\left\langle u_{k}, \tilde{m}_{k}\right\rangle=0,
\end{aligned}
$$

so $f \perp\left[\mathcal{M}_{0}\right]$. This implies that:

$$
f \in \mathcal{M} \Leftrightarrow f \in \mathcal{M} \ominus\left[\mathcal{M}_{0}\right]=\mathcal{N} .
$$

Thus, we have the following equivalent relation: 


$$
f \in \mathcal{N} \Leftrightarrow f \perp K_{1} .
$$

Step 2. It is obvious that $f \perp K_{1}$ is equivalent to

$$
\left\langle\sum_{n=0}^{\infty} z^{n} u_{n},(z-w) z^{i} w^{j}\right\rangle=0, \quad \forall i, j \geq 0 .
$$

And it is easy to verify that:

$$
\begin{aligned}
0 & =\left\langle\sum_{n=0}^{\infty} z^{n} u_{n},(z-w) z^{i} w^{j}\right\rangle \\
& =\sum_{n=0}^{i+1}\left\langle z^{n} u_{n}, z^{i+1} w^{j}\right\rangle-\sum_{n=0}^{i}\left\langle z^{n} u_{n}, z^{i} w^{j+1}\right\rangle \\
& =\left\langle u_{i+1}, w^{j}\right\rangle+\sum_{n=0}^{i}\left\langle z^{n} u_{n},(z-w) z^{i} w^{j}\right\rangle .
\end{aligned}
$$

Since $f_{n}^{0} \perp K_{1}$, so we get:

$$
\sum_{n=0}^{i}\left\langle z^{n} u_{n},(z-w) z^{i} w^{j}\right\rangle=\sum_{n=0}^{i}\left\langle f_{n},(z-w) z^{i-n} w^{j}\right\rangle .
$$

Note that $f_{n} \perp z K_{1}$ and $(z-w) z^{i-n} w^{j} \in z K_{1}(n<i)$, we have:

$$
\sum_{n=0}^{i}\left\langle f_{n},(z-w) z^{i-n} w^{j}\right\rangle=\left\langle f_{i},(z-w) w^{j}\right\rangle .
$$

Hence, $f \in \mathcal{N}$ is equivalent to:

$$
\left\langle u_{i+1}, w^{j}\right\rangle+\left\langle f_{i},(z-w) w^{j}\right\rangle=0, \quad \forall i, j \geq 0 .
$$

Step 3. When $j=0$, since $1 \in N_{1}$, so $1 \perp f_{n}(n \geq 0)$, thus, we get:

$$
\left\langle u_{i+1}, 1\right\rangle=\left\langle f_{i+1}^{0}, 1\right\rangle=\left\langle S_{z} h_{i+1}, 1\right\rangle=\left\langle h_{i+1}, S_{z}^{*} 1\right\rangle=0 .
$$

On the other hand, it is easy to verify that:

$$
\left\langle f_{i}, z-w\right\rangle=\left\langle\frac{C_{0}^{i}}{\sqrt{2}}(z-w), z-w\right\rangle=\sqrt{2} C_{0}^{i} .
$$

Then, in this case, (3.13) is equivalent to $C_{0}^{i}=0(i \geq 0)$.

Step 4 . When $j \geq 1$, it is easy to verify that: 


$$
\begin{aligned}
\left\langle f_{i},(z-w) w^{j}\right\rangle & =\left\langle f_{i}, z w^{j}\right\rangle-\left\langle f_{i}, w^{j+1}\right\rangle \\
& =\frac{j+2}{\sqrt{(j+1)(j+2)}} C_{j}^{i},
\end{aligned}
$$

and

$$
\begin{aligned}
\left\langle u_{i+1}, w^{j}\right\rangle & =\left\langle f_{i+1}^{0}, w^{j}\right\rangle+\left\langle f_{i+1}^{0}, w^{j}\right\rangle \\
& =\frac{1}{\sqrt{j+1}}\left\langle f_{i+1}^{0}, \tilde{e}_{j}\right\rangle-\frac{\sqrt{j}}{\sqrt{j+1}} C_{j-1}^{i+1} .
\end{aligned}
$$

Then, in this case, (3.13) is equivalent to (ii).

Theorem 3.8 Let $\mathcal{M} \subset N_{1}$ and $\mathcal{M} \in \operatorname{Lat}\left(S_{z}\right)$, then $\mathcal{M} \neq\left[\mathcal{M} \ominus S_{z} \mathcal{M}\right]$ if and only if there exists a nonzero solution for the following equations of $C_{k}^{i}(k \geq 0, i \geq 0)$ :

(i) $C_{0}^{i}=0(i \geq 0)$;

(ii) $\left\langle f_{i+1}^{0}, \tilde{e}_{k}\right\rangle-\sqrt{k} C_{k-1}^{i+1}+\sqrt{k+2} C_{k}^{i}=0, \quad \forall i \geq 0, k \geq 1$;

(iii) $\sum_{i=0}^{\infty} \sum_{k=0}^{\infty}\left|C_{k}^{i}\right|^{2}<\infty$,

where

$$
\begin{aligned}
& f_{i}=\sum_{k=0}^{\infty} \frac{C_{k}^{i}}{\sqrt{k+2}}\left(\frac{\sum_{t=0}^{k} z^{t+1} w^{k-t}}{\sqrt{k+1}}-\sqrt{k+1} w^{k+1}\right), \\
& f_{i}^{0}=-S_{z}\left(\mathcal{B}_{\mathcal{M}}^{*} S_{z}\right)^{-1} P_{\mathcal{M}} T_{z}^{*} f_{i}
\end{aligned}
$$

for any $i \geq 0$, and $\tilde{e}_{0}, \tilde{e}_{1}, \ldots$ is an orthonormal basis of $N_{1}$ as mentioned above.

Proof If $\mathcal{M} \neq\left[\mathcal{M} \ominus S_{z} \mathcal{M}\right]$, then there exists $0 \neq f \in \mathcal{N}=\mathcal{M} \ominus\left[\mathcal{M} \ominus S_{z} \mathcal{M}\right]$. By the above statements, let $f=\sum_{n=0}^{\infty} z^{n} u_{n}$, where

$$
u_{n}=-S_{z}\left(\mathcal{B}_{\mathcal{M}}^{*} S_{z}\right)^{-1} P_{\mathcal{M}} T_{z}^{*} f_{n}+f_{n} \in \mathcal{M}_{00}, f_{n} \in K_{1} \ominus z K_{1} .
$$

By Lemma 3.3, there exists an unique sequence $\left\{C_{k}^{i}\right\}_{k, i \geq 0}$, such that:

$$
\sum_{k=0}^{\infty}\left|C_{k}^{i}\right|^{2}<\infty, \quad \forall i \geq 0
$$

and

$$
f_{i}=\sum_{k=0}^{\infty} \frac{C_{k}^{i}}{\sqrt{k+2}}\left(\frac{\sum_{t=0}^{k} z^{t+1} w^{k-t}}{\sqrt{k+1}}-\sqrt{k+1} w^{k+1}\right), \quad i \geq 0 .
$$

By Lemma 3.7, (i)(ii) hold. Note that $\left\langle f_{i}^{0}, f_{i}\right\rangle=0$ for any $i \geq 0$ and there exists $C>0$ (depending on $\mathcal{M}$ ) such that: 


$$
\left\|f_{i}^{0}\right\| \leq C\left\|f_{i}\right\|, \quad i \geq 0
$$

then

$$
\begin{aligned}
\sum_{n=0}^{\infty}\left\|u_{n}\right\|^{2} & =\sum_{n=0}^{\infty}\left\|f_{n}^{0}\right\|^{2}+\sum_{n=0}^{\infty}\left\|f_{n}\right\|^{2}<\infty \\
& \Leftrightarrow \sum_{n=0}^{\infty}\left\|f_{n}\right\|^{2}<\infty \Leftrightarrow \sum_{i=0}^{\infty} \sum_{k=0}^{\infty}\left|C_{k}^{i}\right|^{2}<\infty .
\end{aligned}
$$

Since $f \neq 0$, i.e. $\left\{C_{k}^{i}\right\}_{k, i \geq 0}$ are not completely zero, so $\left\{C_{k}^{i}\right\}_{k, i \geq 0}$ is a nonzero solution for the above equations.

Conversely, assume $\left\{C_{k}^{i}\right\}_{k, i \geq 0}$ is a nonzero solution for the above equations. Let:

$$
f_{i}=\sum_{k=0}^{\infty} \frac{C_{k}^{i}}{\sqrt{k+2}}\left(\frac{\sum_{t=0}^{k} z^{t+1} w^{k-t}}{\sqrt{k+1}}-\sqrt{k+1} w^{k+1}\right), i \geq 0,
$$

then $\left\{f_{i}\right\}_{i \geq 0} \subset K_{1} \ominus z K_{1}$. Let

$$
f_{i}^{0}=-S_{z}\left(\mathcal{B}_{\mathcal{M}}^{*} S_{z}\right)^{-1} P_{\mathcal{M}} T_{z}^{*} f_{i}, i \geq 0 .
$$

By Theorem 3.5, $u_{n}=f_{n}^{0}+f_{n} \in \mathcal{M}_{00}$ for all $n \geq 0$. Let $f=\sum_{n=0}^{\infty} z^{n} u_{n} \in \tilde{\mathcal{N}}$, by Lemma 3.7 again, we have $0 \neq f \in \mathcal{N}=\mathcal{M} \ominus\left[\mathcal{M} \ominus S_{z} \mathcal{M}\right]$. This implies $\mathcal{M} \neq\left[\mathcal{M} \ominus S_{z} \mathcal{M}\right]$.

As we know, the Beurling type theorem holds for $S_{z}$ on $N_{1}$, thus we have the following corollary through Theorem 3.8 which reflect the common property of invariant subspaces of $S_{z}$.

Corollary 3.9 Let $\mathcal{M} \subset N_{1}$ and $\mathcal{M} \in \operatorname{Lat}\left(S_{z}\right)$, then the following equations of $C_{k}^{i}(k \geq 0, i \geq 0)$ has only zero solution:

(i) $C_{0}^{i}=0(i \geq 0)$;

(ii) $\left\langle f_{i+1}^{0}, \tilde{e}_{k}\right\rangle-\sqrt{k} C_{k-1}^{i+1}+\sqrt{k+2} C_{k}^{i}=0, \quad \forall i \geq 0, k \geq 1$;

(iii) $\sum_{i=0}^{\infty} \sum_{k=0}^{\infty}\left|C_{k}^{i}\right|^{2}<\infty$,

where

$$
\begin{aligned}
f_{i} & =\sum_{k=0}^{\infty} \frac{C_{k}^{i}}{\sqrt{k+2}}\left(\frac{\sum_{t=0}^{k} z^{t+1} w^{k-t}}{\sqrt{k+1}}-\sqrt{k+1} w^{k+1}\right), \\
f_{i}^{0} & =-S_{z}\left(\mathcal{B}_{\mathcal{M}}^{*} S_{z}\right)^{-1} P_{\mathcal{M}} T_{z}^{*} f_{i}
\end{aligned}
$$

for any $i \geq 0$, and $\tilde{e}_{0}, \tilde{e}_{1}, \ldots$ is an orthonormal basis of $N_{1}$ as mentioned above. 


\section{The proof of Theorem 1.8}

Let:

$$
E_{k}(z)=\sqrt{k+1} z^{k}, \quad \forall k \geq 0,
$$

then $\left\{E_{k}\right\}_{k \geq 0}$ is an orthonormal basis of $L_{a}^{2}$. For any fixed $a \in \mathbb{D}$, the reproducing kernel of $L_{a}^{2}$ is

$$
K_{a}(z)=\frac{1}{(1-\bar{a} z)^{2}}=\sum_{n=0}^{\infty} \overline{E_{n}(a)} E_{n}(z) .
$$

Define the operator $U: L_{a}^{2} \rightarrow N_{1}$ such that

$$
U E_{k}=\tilde{e}_{k}, \quad \forall k \geq 0 .
$$

Lemma 4.1 $U$ is a unitary operator and $S_{z} U=U \mathcal{B}$, that is, $S_{z}$ and $\mathcal{B}$ are unitarily equivalent.

Proof Since,

$$
S_{z} \tilde{e}_{k}=\frac{\sqrt{k+1}}{\sqrt{k+2}} \tilde{e}_{k+1}, \quad \forall k \geq 0,
$$

and

$$
\mathcal{B} E_{k}=\sqrt{k+1} z^{k+1}=\frac{\sqrt{k+1}}{\sqrt{k+2}} E_{k+1}, \quad \forall k \geq 0,
$$

we get the desired result.

Remark 4.2 For any fixed $a \in \mathbb{D}$, let $\mathcal{M}_{a}=U I_{a}$, then $\mathcal{M}_{a} \subset N_{1}$ and $\mathcal{M}_{a}$ is an invariant subspace of $S_{z}$. Let $P_{\mathcal{M}_{a}}: N_{1} \rightarrow \mathcal{M}_{a}$ be the projection operator. For any $g$ in $N_{1}$, let $g=\sum_{n=0}^{\infty} \alpha_{n} \tilde{e}_{n}$, then,

$$
\begin{aligned}
\left(I-P_{\mathcal{M}_{a}}\right) g & =\left\langle\left(I-P_{\mathcal{M}_{a}}\right) g, \frac{U K_{a}}{\left\|K_{a}\right\|}\right\rangle \frac{U K_{a}}{\left\|K_{a}\right\|} \\
& =\left\langle g, \frac{U K_{a}}{\left\|K_{a}\right\|}\right\rangle \frac{U K_{a}}{\left\|K_{a}\right\|},
\end{aligned}
$$

that is:

$$
P_{\mathcal{M}_{a}} g=g-\left(1-|a|^{2}\right)^{2}\left\langle g, U K_{a}\right\rangle U K_{a},
$$

where 


$$
U K_{a}=\sum_{n=0}^{\infty} \overline{E_{n}(a)} \tilde{e}_{n}
$$

Proposition 4.3 For $a \in \mathbb{D}$, we have:

$$
\begin{aligned}
\left(\mathcal{B}_{\mathcal{M}_{a}}^{*} S_{z}\right)^{-1} P_{\mathcal{M}_{a}} \tilde{e}_{k} \\
=-\frac{k+2}{(k+1)\left(2-|a|^{2}\right)}\left(1-|a|^{2}\right)^{2} E_{k}(a) \sum_{n=0}^{\infty} \frac{n+2}{n+1} \overline{E_{n}(a)} \tilde{e}_{n} \\
\quad+\frac{k+2}{k+1} \tilde{e}_{k}, \quad \forall k \geq 0 .
\end{aligned}
$$

Proof For any fixed $k \geq 0$, let: $f=\left(\mathcal{B}_{\mathcal{M}_{a}}^{*} S_{z}\right)^{-1} P_{\mathcal{M}_{a}} \tilde{e}_{k}$, then there exists $g=\sum_{n=0}^{\infty} \alpha_{n} \tilde{e}_{n} \in N_{1}$ such that $f=P_{\mathcal{M}_{a}} g$, i.e., $\mathcal{B}_{\mathcal{M}_{a}}^{*} S_{z} P_{\mathcal{M}_{a}} g=P_{\mathcal{M}_{a}} \tilde{e}_{k}$. By (4.2), we get:

$$
P_{\mathcal{M}_{a}} \tilde{e}_{k}=\tilde{e}_{k}-\left(1-|a|^{2}\right)^{2} E_{k}(a) \sum_{n=0}^{\infty} \overline{E_{n}(a)} \tilde{e}_{n}
$$

Since:

$$
S_{z}^{*} S_{z} \tilde{e}_{k}=\frac{k+1}{k+2} \tilde{e}_{k}, \quad \forall k \geq 0
$$

then,

$$
\begin{aligned}
S_{z}^{*} S_{z} f= & \sum_{n=0}^{\infty} \alpha_{n} \frac{n+1}{n+2} \tilde{e}_{n}-\left(1-|a|^{2}\right)^{2} \\
& \times\left(\sum_{m=0}^{\infty} \alpha_{m} E_{m}(a)\right) \sum_{n=0}^{\infty} \overline{E_{n}(a)} \frac{n+1}{n+2} \tilde{e}_{n} \\
= & \sum_{n=0}^{\infty} \frac{n+1}{n+2}\left[\alpha_{n}-\left(1-|a|^{2}\right)^{2}\left(\sum_{m=0}^{\infty} \alpha_{m} E_{m}(a)\right) \overline{E_{n}(a)}\right] \tilde{e}_{n} .
\end{aligned}
$$

For convenience, let:

$$
\beta_{n}=\frac{n+1}{n+2}\left[\alpha_{n}-\left(1-|a|^{2}\right)^{2}\left(\sum_{m=0}^{\infty} \alpha_{m} E_{m}(a)\right) \overline{E_{n}(a)}\right], \quad \forall n \geq 0,
$$

then,

$$
f=\sum_{n=0}^{\infty} \frac{n+2}{n+1} \beta_{n} \tilde{e}_{n}, \quad S_{z}^{*} S_{z} f=\sum_{n=0}^{\infty} \beta_{n} \tilde{e}_{n}
$$

and, 


$$
\mathcal{B}_{\mathcal{M}_{a}}^{*} S_{z} f=\sum_{n=0}^{\infty}\left[\beta_{n}-\left(1-|a|^{2}\right)^{2}\left(\sum_{m=0}^{\infty} \beta_{m} E_{m}(a)\right) \overline{E_{n}(a)}\right] \tilde{e}_{n} .
$$

Hence, $\mathcal{B}_{\mathcal{M}_{a}}^{*} S_{z} P_{\mathcal{M}_{a}} g=P_{\mathcal{M}_{a}} \tilde{e}_{k}$ if and only if the following equations hold:

$$
\beta_{k}=1-\left(1-|a|^{2}\right)^{2}\left|E_{k}(a)\right|^{2}+\left(1-|a|^{2}\right)^{2}\left(\sum_{m=0}^{\infty} \beta_{m} E_{m}(a)\right) \overline{E_{k}(a)},
$$

and

$$
\begin{aligned}
\beta_{n}= & -\left(1-|a|^{2}\right)^{2} E_{k}(a) \overline{E_{n}(a)} \\
& +\left(1-|a|^{2}\right)^{2}\left(\sum_{m=0}^{\infty} \beta_{m} E_{m}(a)\right) \overline{E_{n}(a)}, \quad n \geq 0, n \neq k
\end{aligned}
$$

Let:

$$
B_{a}=\sum_{m=0}^{\infty} \beta_{m} E_{m}(a)
$$

since by Eqs. (4.9) and (4.10), we have:

$$
\begin{aligned}
\sum_{n=0}^{\infty} \beta_{n} E_{n}(a)= & E_{k}(a)-\left(1-|a|^{2}\right)^{2} E_{k}(a) \sum_{n=0}^{\infty}\left|E_{n}(a)\right|^{2} \\
& +\left(1-|a|^{2}\right)^{2} B_{a} \sum_{n=0}^{\infty}\left|E_{n}(a)\right|^{2}=B_{a},
\end{aligned}
$$

this shows that Eq. (4.11) is compatible with Eqs. (4.9) and (4.10).

Let:

$$
A_{a}=\sum_{m=0}^{\infty} \alpha_{m} E_{m}(a)
$$

then by (4.7),(4.9) and (4.10), we get

$$
\begin{aligned}
\alpha_{k}= & \frac{k+2}{k+1}+\frac{k+2}{k+1}\left[-\left(1-|a|^{2}\right)^{2}\left|E_{k}(a)\right|^{2}\right. \\
& \left.+\left(1-|a|^{2}\right)^{2}\left(\sum_{m=0}^{\infty} \beta_{m} E_{m}(a)\right) \overline{E_{k}(a)}\right]+\left(1-|a|^{2}\right)^{2} A_{a} \overline{E_{k}(a)},
\end{aligned}
$$

and 


$$
\begin{aligned}
\alpha_{n}= & \frac{n+2}{n+1}\left[-\left(1-|a|^{2}\right)^{2} E_{k}(a) \overline{E_{n}(a)}\right. \\
& \left.+\left(1-|a|^{2}\right)^{2}\left(\sum_{m=0}^{\infty} \beta_{m} E_{m}(a)\right) \overline{E_{n}(a)}\right] \\
& +\left(1-|a|^{2}\right)^{2} A_{a} \overline{E_{n}(a)}, \quad n \geq 0, n \neq k .
\end{aligned}
$$

And one calculates that:

$$
\begin{aligned}
\sum_{n=0}^{\infty} \alpha_{n} E_{n}(a)= & \frac{k+2}{k+1} E_{k}(a)+\sum_{n=0}^{\infty} \frac{n+2}{n+1}\left[-\left(1-|a|^{2}\right)^{2} E_{k}(a) \overline{E_{n}(a)}\right. \\
& \left.+\left(1-|a|^{2}\right)^{2}\left(\sum_{m=0}^{\infty} \beta_{m} E_{m}(a)\right) \overline{E_{n}(a)}\right] E_{n}(a) \\
& +\left(1-|a|^{2}\right)^{2} A_{a} \sum_{n=0}^{\infty}\left|E_{n}(a)\right|^{2} \\
= & \frac{k+2}{k+1} E_{k}(a)+A_{a} \\
& -\left(1-|a|^{2}\right)^{2} E_{k}(a) \sum_{n=0}^{\infty} \frac{n+2}{n+1}\left|E_{n}(a)\right|^{2} \\
& +\left(1-|a|^{2}\right)^{2} B_{a} \sum_{n=0}^{\infty} \frac{n+2}{n+1}\left|E_{n}(a)\right|^{2}
\end{aligned}
$$

since,

$$
\begin{aligned}
& \sum_{n=0}^{\infty} \frac{n+2}{n+1}\left|E_{n}(a)\right|^{2}=\sum_{n=0}^{\infty}(n+1)|a|^{2 n}+\sum_{n=0}^{\infty}|a|^{2 n} \\
& \quad=\sum_{n=0}^{\infty} \frac{\Gamma(n+2)}{n ! \Gamma(2)} a^{n} \bar{a}^{n}+\frac{1}{1-|a|^{2}}=\frac{1}{\left(1-|a|^{2}\right)^{2}}+\frac{1}{1-|a|^{2}},
\end{aligned}
$$

(since, $\left.\frac{1}{(1-z \bar{w})^{2+\alpha}}=\sum_{n=0}^{\infty} \frac{\Gamma(n+2+\alpha)}{n ! \Gamma(2+\alpha)} z^{n} \bar{w}^{n}\right)$ then,

$$
\begin{aligned}
A_{a}= & \sum_{n=0}^{\infty} \alpha_{n} E_{n}(a)=\frac{k+2}{k+1} E_{k}(a)+A_{a} \\
& -\left(1-|a|^{2}\right)^{2} E_{k}(a)\left(\frac{1}{\left(1-|a|^{2}\right)^{2}}+\frac{1}{1-|a|^{2}}\right) \\
& +\left(1-|a|^{2}\right)^{2} B_{a}\left(\frac{1}{\left(1-|a|^{2}\right)^{2}}+\frac{1}{1-|a|^{2}}\right),
\end{aligned}
$$

that is equivalent to: 


$$
\begin{aligned}
0= & \frac{k+2}{k+1} E_{k}(a)-\left(1-|a|^{2}\right)^{2} E_{k}(a)\left(\frac{1}{\left(1-|a|^{2}\right)^{2}}+\frac{1}{1-|a|^{2}}\right) \\
& +\left(1-|a|^{2}\right)^{2} B_{a}\left(\frac{1}{\left(1-|a|^{2}\right)^{2}}+\frac{1}{1-|a|^{2}}\right) .
\end{aligned}
$$

Then,

$$
B_{a}=\left(1-\frac{\frac{k+2}{k+1}}{2-|a|^{2}}\right) E_{k}(a)
$$

Hence,

$$
\beta_{k}=1-\left(1-|a|^{2}\right)^{2}\left|E_{k}(a)\right|^{2}+\left(1-|a|^{2}\right)^{2}\left(1-\frac{\frac{k+2}{k+1}}{2-|a|^{2}}\right)\left|E_{k}(a)\right|^{2},
$$

and,

$$
\begin{aligned}
\beta_{m}= & -\left(1-|a|^{2}\right)^{2} E_{k}(a) \overline{E_{m}(a)} \\
& +\left(1-|a|^{2}\right)^{2}\left(1-\frac{\frac{k+2}{k+1}}{2-|a|^{2}}\right) E_{k}(a) \overline{E_{m}(a)}, \quad m \geq 0, m \neq k
\end{aligned}
$$

By the above calculates, we can choose $A_{a}$ being any positive constant, such that:

$$
\begin{aligned}
\alpha_{m}= & \frac{m+2}{m+1}\left[-E_{k}(a)+\left(1-\frac{\frac{k+2}{k+1}}{2-|a|^{2}}\right) E_{k}(a)+A_{a}\right]\left(1-|a|^{2}\right)^{2} \overline{E_{m}(a)} \\
= & \frac{m+2}{m+1}\left[-\frac{k+2}{(k+1)\left(2-|a|^{2}\right)} E_{k}(a)\right. \\
& \left.+A_{a}\right]\left(1-|a|^{2}\right)^{2} \overline{E_{m}(a)}, \quad m \neq k, m \geq 0 .
\end{aligned}
$$

Note that:

$$
\sum_{m=k+1}^{\infty}\left|\alpha_{m}\right|^{2} \leq C_{a, k} \sum_{m=0}^{\infty}(m+1)|a|^{2 m}=\frac{C_{a, k}}{\left(1-|a|^{2}\right)^{2}}<\infty,
$$

where $C_{a, k}$ is a positive constant depending only on $a, k$. Then, 


$$
\begin{aligned}
f= & \sum_{n=0}^{\infty} \frac{n+2}{n+1} \beta_{n} \tilde{e}_{n} \\
= & -\frac{k+2}{(k+1)\left(2-|a|^{2}\right)}\left(1-|a|^{2}\right)^{2} E_{k}(a) \\
& \times \sum_{n=0}^{\infty} \frac{n+2}{n+1} \overline{E_{n}(a)} \tilde{e}_{n}+\frac{k+2}{k+1} \tilde{e}_{k} \\
= & \left(\mathcal{B}_{\mathcal{M}_{a}}^{*} S_{z}\right)^{-1} P_{\mathcal{M}_{a}} \tilde{e}_{k}, \quad \forall k \geq 0 .
\end{aligned}
$$

Theorem 4.4 If $a \in \mathbb{D}$, then $I_{a} \neq\left[I_{a} \ominus \mathcal{B} I_{a}\right]$ holds in $L_{a}^{2}$ if and only if there exists $a$ nonzero solution for the following equations of $C_{1}^{i}(i \geq 0)$ :

(I) $\quad \sum_{n=1}^{\infty}\left(\sum_{j=0}^{n-1} \bar{a}^{n-1-j} C_{1}^{i+1+j}\right) a^{n}=-\frac{2-|a|^{2}}{\left(1-|a|^{2}\right)^{2}} C_{1}^{i}, \quad \forall i \geq 0$;

(II) $\sum_{i=0}^{\infty} \sum_{k=1}^{\infty}\left|C_{k}^{i}\right|^{2}<\infty$,

where

$$
C_{k}^{i}=\frac{\sqrt{3}}{\sqrt{2}} \frac{\sqrt{k+1}}{\sqrt{k+2}} \sum_{j=0}^{k-1} \bar{a}^{k-1-j} C_{1}^{i+j}, \quad \forall k \geq 2, i \geq 0 .
$$

Proof For any fixed $a \in \mathbb{D}$, let $\mathcal{M}=\mathcal{M}_{a}$ in Theorem 3.8, we easily calculate that:

$$
\begin{aligned}
\left\langle f_{i+1}^{0}, \tilde{e}_{k}\right\rangle & =-\left\langle S_{z}\left(\mathcal{B}_{\mathcal{M}_{a}}^{*} S_{z}\right)^{-1} P_{\mathcal{M}_{a}} T_{z}^{*} f_{i+1}, \tilde{e}_{k}\right\rangle \\
& =-\left\langle T_{z}^{*} f_{i+1},\left(\mathcal{B}_{\mathcal{M}_{a}}^{*} S_{z}\right)^{-1} P_{\mathcal{M}_{a}} S_{z}^{*} \tilde{e}_{k}\right\rangle,
\end{aligned}
$$

and

$$
\begin{gathered}
S_{z}^{*} \tilde{e}_{0}=0, \quad S_{z}^{*} \tilde{e}_{k}=\frac{\sqrt{k}}{\sqrt{k+1}} \tilde{e}_{k-1}, \quad \forall k \geq 1 ; \\
T_{z}^{*} f_{i}=\sum_{n=0}^{\infty} \frac{C_{n}^{i}}{\sqrt{n+2}} \tilde{e}_{n}, \quad \forall i \geq 0 .
\end{gathered}
$$

Then, 


$$
\begin{aligned}
\left\langle f_{i+1}^{0}, \tilde{e}_{0}\right\rangle= & 0, \\
\left\langle f_{i+1}^{0}, \tilde{e}_{k}\right\rangle= & -\frac{\sqrt{k}}{\sqrt{k+1}}\left\langle\sum_{n=0}^{\infty} \frac{C_{n}^{i+1}}{\sqrt{n+2}} \tilde{e}_{n},\left(\mathcal{B}_{\mathcal{M}_{a}}^{*} S_{z}\right)^{-1} P_{\mathcal{M}_{a}} \tilde{e}_{k-1}\right\rangle \\
= & -\frac{\sqrt{k}}{\sqrt{k+1}}\left\langle\sum_{n=0}^{\infty} \frac{C_{n}^{i+1}}{\sqrt{n+2}} \tilde{e}_{n},-\frac{k+1}{k\left(2-|a|^{2}\right)}\left(1-|a|^{2}\right)^{2} E_{k-1}(a)\right. \\
& \left.\times \sum_{n=0}^{\infty} \frac{n+2}{n+1} \overline{E_{n}(a)} \tilde{e}_{n}+\frac{k+1}{k} \tilde{e}_{k-1}\right\rangle \\
= & \frac{\sqrt{k+1}}{\sqrt{k}\left(2-|a|^{2}\right)}\left(1-|a|^{2}\right)^{2} \frac{E_{k-1}(a)}{\sum_{n=0}^{\infty}} \frac{\sqrt{n+2}}{n+1} C_{n}^{i+1} E_{n}(a)-\frac{C_{k-1}^{i+1}}{\sqrt{k}} \\
= & \frac{\left(1-|a|^{2}\right)^{2}}{2-|a|^{2}} \sqrt{k+1} \bar{a}^{k-1} \sum_{n=0}^{\infty} \frac{\sqrt{n+2}}{\sqrt{n+1}} C_{n}^{i+1} a^{n}-\frac{C_{k-1}^{i+1}}{\sqrt{k}}, \quad \forall k \geq 1 .
\end{aligned}
$$

Then (ii) in Theorem 3.8 is:

$$
\begin{aligned}
& \frac{\left(1-|a|^{2}\right)^{2}}{2-|a|^{2}} \sqrt{k+1} \bar{a}^{k-1} \sum_{n=0}^{\infty} \frac{\sqrt{n+2}}{\sqrt{n+1}} C_{n}^{i+1} a^{n} \\
& -\left(\frac{1}{\sqrt{k}}+\sqrt{k}\right) C_{k-1}^{i+1}+\sqrt{k+2} C_{k}^{i}=0, \quad \forall i \geq 0, k \geq 1 .
\end{aligned}
$$

If $C_{0}^{i}=0(i \geq 0)$, let $k=1$ in (4.27), we get:

$$
\frac{\sqrt{2}\left(1-|a|^{2}\right)^{2}}{2-|a|^{2}} \sum_{n=1}^{\infty} \frac{\sqrt{n+2}}{\sqrt{n+1}} C_{n}^{i+1} a^{n}=-\sqrt{3} C_{1}^{i} .
$$

Hence, (4.27) is equivalent to holding the following equations:

$$
\begin{gathered}
\sum_{n=1}^{\infty} \frac{\sqrt{n+2}}{\sqrt{n+1}} C_{n}^{i+1} a^{n}=-\frac{\sqrt{3}\left(2-|a|^{2}\right)}{\sqrt{2}\left(1-|a|^{2}\right)^{2}} C_{1}^{i}, \quad \forall i \geq 0 \\
-\frac{\sqrt{3}}{\sqrt{2}} \sqrt{k+1} \bar{a}^{k-1} C_{1}^{i}-\frac{1+k}{\sqrt{k}} C_{k-1}^{i+1}+\sqrt{k+2} C_{k}^{i}=0, \quad \forall i \geq 0, k \geq 2 .
\end{gathered}
$$

By the induction, we easily get that Eq. (4.30) is equivalent to the following equations:

$$
C_{k}^{i}=\frac{\sqrt{3}}{\sqrt{2}} \frac{\sqrt{k+1}}{\sqrt{k+2}} \sum_{j=0}^{k-1} \bar{a}^{k-1-j} C_{1}^{i+j}, \quad \forall k \geq 2, i \geq 0 .
$$


Then, Theorem 3.8 implies that $\mathcal{M}_{a} \neq\left[\mathcal{M}_{a} \ominus S_{z} \mathcal{M}_{a}\right]$ holds in $N_{1}$ if and only if there exists a nonzero solution for the following equations of $C_{1}^{i}(i \geq 0)$ :

$$
\begin{aligned}
& \text { (I) } \sum_{n=1}^{\infty}\left(\sum_{j=0}^{n-1} \bar{a}^{n-1-j} C_{1}^{i+1+j}\right) a^{n}=-\frac{2-|a|^{2}}{\left(1-|a|^{2}\right)^{2}} C_{1}^{i}, \quad \forall i \geq 0 ; \\
& \text { (II) } \sum_{i=0}^{\infty} \sum_{k=1}^{\infty}\left|C_{k}^{i}\right|^{2}<\infty,
\end{aligned}
$$

where

$$
C_{k}^{i}=\frac{\sqrt{3}}{\sqrt{2}} \frac{\sqrt{k+1}}{\sqrt{k+2}} \sum_{j=0}^{k-1} \bar{a}^{k-1-j} C_{1}^{i+j}, \quad \forall k \geq 2, i \geq 0 .
$$

And Lemma 4.1 implies that: $I_{a} \neq\left[I_{a} \ominus \mathcal{B} I_{a}\right]$ holds in $L_{a}^{2}$ if and only if $\mathcal{M}_{a} \neq\left[\mathcal{M}_{a} \ominus S_{z} \mathcal{M}_{a}\right]$ holds in $N_{1}$, so we get the desired result.

Proof of Theorem 1.8 For any $0 \neq a \in \mathbb{D}$, by Theorem 4.4, we get that $I_{a} \neq\left[I_{a} \ominus \mathcal{B} I_{a}\right]$ holds in $L_{a}^{2}$ if and only if the following equations of $C_{1}^{i}(i \geq 0)$ :

$$
\sum_{n=1}^{\infty}\left(\sum_{j=0}^{n-1} \bar{a}^{n-1-j} C_{1}^{i+1+j}\right) a^{n}=-\frac{2-|a|^{2}}{\left(1-|a|^{2}\right)^{2}} C_{1}^{i}, \quad \forall i \geq 0,
$$

under the condition (II) in Theorem 4.4 has a nonzero solution. For any $i \geq 0$, let:

$$
\begin{aligned}
& x_{n m}=\bar{a}^{n-1-m} C_{1}^{i+1+m} a^{n}, \text { when } 0 \leq m \leq n-1, n \geq 1 ; \\
& x_{n m}=0, \text { when } 0 \leq n-1<m .
\end{aligned}
$$

Then,

$$
\sum_{n=1}^{\infty}\left(\sum_{j=0}^{n-1} \bar{a}^{n-1-j} C_{1}^{i+1+j}\right) a^{n}=\sum_{n=1}^{\infty} \sum_{m=0}^{n-1} x_{n m}
$$

Note that:

$$
\begin{aligned}
\sum_{n=1}^{\infty} \sum_{m=0}^{\infty}\left|x_{n m}\right| & =\sum_{n=1}^{\infty} \sum_{m=0}^{n-1}\left|x_{n m}\right|=\sum_{n=1}^{\infty}|a|^{2 n-1} \sum_{m=0}^{n-1}\left(\frac{1}{|a|}\right)^{m}\left|C_{1}^{i+1+m}\right| \\
& \leq \sum_{n=1}^{\infty}|a|^{2 n-1}\left(\sum_{m=0}^{n-1}\left(\frac{1}{|a|}\right)^{2 m}\right)^{\frac{1}{2}}\left(\sum_{m=0}^{\infty}\left|C_{1}^{m}\right|^{2}\right)^{\frac{1}{2}} \\
& \leq C \sum_{n=1}^{\infty}|a|^{2 n-1}\left(\frac{|a|^{2}\left(\frac{1}{|a|^{2 n}}-1\right)}{1-|a|^{2}}\right)^{\frac{1}{2}} \\
& \leq C \frac{|a|}{\sqrt{1-|a|^{2}}} \sum_{n=1}^{\infty}|a|^{2 n-1} \frac{1}{|a|^{n}} \\
& =C \frac{|a|}{(1-|a|) \sqrt{1-|a|^{2}}}<+\infty,
\end{aligned}
$$


where $C$ is a positive constant, so we have:

$$
\begin{aligned}
\sum_{n=1}^{\infty} \sum_{m=0}^{\infty} x_{n m} & =\sum_{m=0}^{\infty} \sum_{n=1}^{\infty} x_{n m}=\sum_{m=0}^{\infty} \sum_{n=m+1}^{\infty} x_{n m} \\
& =\sum_{m=0}^{\infty} \sum_{n=m+1}^{\infty} \bar{a}^{n-1-m} C_{1}^{i+1+m} a^{n} \\
& =\sum_{m=1}^{\infty} C_{1}^{i+m} \frac{1}{\bar{a}^{m}}\left(\sum_{n=m}^{\infty}|a|^{2 n}\right) \\
& =\frac{1}{1-|a|^{2}} \sum_{m=1}^{\infty} a^{m} C_{1}^{i+m} .
\end{aligned}
$$

Then, (4.32) is equivalent to the following form:

$$
\sum_{m=1}^{\infty} a^{m} C_{1}^{i+m}=-\frac{2-|a|^{2}}{1-|a|^{2}} C_{1}^{i}, \quad \forall i \geq 0 .
$$

That is also equivalent to the following matrix form:

$$
\begin{gathered}
\left(\begin{array}{cccccccccc}
0 & a & a^{2} & a^{3} & a^{4} & a^{5} & a^{6} & \cdot & \cdot & \cdot \\
0 & 0 & a & a^{2} & a^{3} & a^{4} & a^{5} & \cdot & . & \cdot \\
0 & 0 & 0 & a & a^{2} & a^{3} & a^{4} & \cdot & \cdot & \cdot \\
0 & 0 & 0 & 0 & a & a^{2} & a^{3} & \cdot & \cdot & \cdot \\
0 & 0 & 0 & 0 & 0 & a & a^{2} & \cdot & \cdot & \cdot \\
\cdot & \cdot & \cdot & & & & & &
\end{array}\right)\left(\begin{array}{c}
C_{1}^{0} \\
C_{1}^{1} \\
C_{1}^{2} \\
C_{1}^{3} \\
C_{1}^{4} \\
\cdot
\end{array}\right) \\
=\lambda_{a}\left(\begin{array}{c}
C_{1}^{0} \\
C_{1}^{1} \\
C_{1}^{2} \\
C_{1}^{3} \\
C_{1}^{4}
\end{array}\right),
\end{gathered}
$$

where

$$
\lambda_{a}=-\frac{2-|a|^{2}}{1-|a|^{2}} .
$$

Since, the matrix: 


$$
A_{a}=\left(\begin{array}{cccccccccc}
0 & a & a^{2} & a^{3} & a^{4} & a^{5} & a^{6} & . & . & . \\
0 & 0 & a & a^{2} & a^{3} & a^{4} & a^{5} & . & . & . \\
0 & 0 & 0 & a & a^{2} & a^{3} & a^{4} & . & . & . \\
0 & 0 & 0 & 0 & a & a^{2} & a^{3} & . & . & . \\
0 & 0 & 0 & 0 & 0 & a & a^{2} & . & . & . \\
. & . & & . & & & & & &
\end{array}\right)
$$

is the matrix of the Toeplitz operator with symbol $\phi\left(e^{i \theta}\right) \in L^{\infty}(\mathbb{T})$ with respect to the basis $\left\{e^{i n \theta}\right\}_{n=0}^{\infty}$ of $H^{2}$, and let $\phi_{k}$ be the kth Fourier coefficient of $\phi\left(e^{i \theta}\right)$, then

$$
\phi_{k}=0, \quad \text { when } k \geq 0 ; \phi_{k}=a^{-k} \text {, when } k<0 .
$$

Thus, we get:

$$
\phi\left(e^{i \theta}\right)=\sum_{k=1}^{\infty} a^{k} e^{-i k \theta}=\frac{1}{1-a e^{-i \theta}}-1 .
$$

Note that (4.36) has a nonzero solution $\left\{C_{1}^{i}\right\}_{i \geq 0}$ such that:

$$
\sum_{i=0}^{\infty}\left|C_{1}^{i}\right|^{2}<+\infty
$$

which is equivalent to $\lambda_{a}$ being an eigenvalue of Toeplitz operator $T_{\phi}\left(f=\sum_{i=0}^{\infty} C_{1}^{i} z^{i}\right.$ is an eigenvector). This completes the proof.

Corollary 4.5 For any $a \in \mathbb{D}, I_{a}=\left[I_{a} \ominus \mathcal{B} I_{a}\right]$ holds in $L_{a}^{2}$.

Proof If $a=0$, then (I) in Theorem 4.4 is $C_{1}^{i}=0, \forall i \geq 0$. If $C_{1}^{i}=0$, then (II) in Theorem 4.4 holds. Hence Theorem 4.4 implies that $I_{0}=\left[I_{0} \ominus \mathcal{B} I_{0}\right]$. By Theorem 1.8, in the following process, we need to prove that $\lambda_{a}$ is not an eigenvalue of Toeplitz operator $T_{\phi}$ for any $0 \neq a \in \mathbb{D}$, where,

$$
\lambda_{a}=-\frac{2-|a|^{2}}{1-|a|^{2}},
$$

and

$$
\phi\left(e^{i \theta}\right)=\frac{1}{1-a e^{-i \theta}}-1 .
$$

Note that:

$$
\bar{\phi}(z)=\frac{1}{1-\bar{a} z}-1 \in H^{\infty},
$$

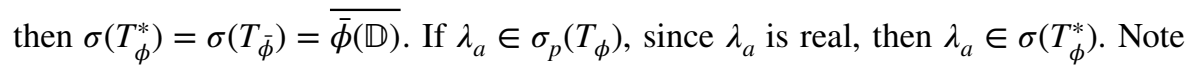
that 


$$
\begin{aligned}
\lambda_{a} & =\bar{\phi}(z) \Leftrightarrow-\left(2-|a|^{2}\right)=\left(1-|a|^{2}\right)\left(\frac{1}{1-\bar{a} z}-1\right) \\
& \Leftrightarrow-(2-b)=(1-b)(x-1) \Leftrightarrow x=\frac{1}{b-1},
\end{aligned}
$$

where $b=|a|^{2}, x=\frac{1}{1-\bar{a} z}$. That is:

$$
\bar{a} z=2-b .
$$

Since $0<b<1$ and $|\bar{a} z|<1$ for all $z \in \mathbb{D}$, then (4.42) fails for any $z \in \overline{\mathbb{D}}$, i.e., $\lambda_{a}-\bar{\phi}(z) \neq 0$ on $\overline{\mathbb{D}}$. But $\lambda_{a}-\bar{\phi}(z)$ is continuous on $\overline{\mathbb{D}}$, hence

$$
\inf _{z \in \mathbb{D}}\left|\lambda_{a}-\bar{\phi}(z)\right| \geq \min _{z \in \overline{\mathbb{D}}}\left|\lambda_{a}-\bar{\phi}(z)\right|>0 .
$$

This implies $\lambda_{a} \notin \overline{\bar{\phi}(\mathbb{D})}$, i.e., $\lambda_{a} \notin \sigma\left(T_{\phi}^{*}\right)$, and this completes the proof.

\section{The proof of Theorem 1.5}

For any fixed $\alpha>-1$, let

$$
E_{n}(z)=\sqrt{\frac{\Gamma(n+\alpha+2)}{n ! \Gamma(\alpha+2)}} z^{n}, \quad \forall n \geq 0,
$$

then $\left\{E_{n}\right\}_{n \geq 0}$ is an orthonormal basis of $L_{a}^{2}\left(d A_{\alpha}\right)$. It is clear that $\mathcal{B}_{\alpha}$ is bounded below and analytic on $L_{a}^{2}\left(d A_{\alpha}\right)$. Let $f=\sum_{n=0}^{\infty} \alpha_{n} E_{n} \in L_{a}^{2}\left(d A_{\alpha}\right)$, then

$$
\mathcal{B}_{\alpha}^{*} f=\sum_{n=0}^{\infty} \beta_{n+1} \frac{\sqrt{n+1}}{\sqrt{n+\alpha+2}} E_{n} .
$$

Lemma 5.1 Let $\mathcal{M}$ be an invariant subspace of $\mathcal{B}_{\alpha}$ in $L_{a}^{2}\left(d A_{\alpha}\right)$. Then $\mathcal{B}_{\alpha, \mathcal{M}}^{*} \mathcal{B}_{\alpha}$ is invertible on $\mathcal{M}$, where the operator $\mathcal{B}_{\alpha, \mathcal{M}}^{*}$ denotes the adjoint of the restriction $\left.\mathcal{B}_{\alpha}\right|_{\mathcal{M}}$, s.t. $\mathcal{B}_{\alpha, \mathcal{M}}^{*} q=P_{\mathcal{M}} \mathcal{B}_{\alpha}^{*} q$ for $q$ in $\mathcal{M}, P_{\mathcal{M}}$ is the projection operator from $L_{a}^{2}\left(d A_{\alpha}\right)$ onto $\mathcal{M}$.

Proof The proof is similar to Lemma 3.4.

Remark 5.2 For any fixed $a \in \mathbb{D}$, and any $g$ in $L_{a}^{2}\left(d A_{\alpha}\right)$, we have 


$$
\begin{aligned}
\left(P_{I_{a}} g\right)(w) & =\left\langle P_{I_{a}} g, K_{a}^{\alpha}(z, w)\right\rangle=\left\langle g, K_{a}^{\alpha}(z, w)\right\rangle \\
& =g(w)-\left(1-|a|^{2}\right)^{\alpha+2} g(a) \frac{1}{(1-\bar{a} w)^{\alpha+2}} \\
& =g(w)-\left(1-|a|^{2}\right)^{\alpha+2} g(a) \sum_{n=0}^{\infty} \overline{E_{n}(a)} E_{n}(w),
\end{aligned}
$$

that is:

$$
P_{I_{a}} g=g-\left(1-|a|^{2}\right)^{\alpha+2} g(a) \sum_{n=0}^{\infty} \overline{E_{n}(a)} E_{n} .
$$

Repeating the same process of the proof of Proposition 4.3, we get the following proposition:

Proposition 5.3 For $a \in \mathbb{D}$, we have:

$$
\begin{aligned}
\left(\mathcal{B}_{\alpha, I_{a}}^{*} \mathcal{B}_{\alpha}\right)^{-1} P_{I_{a}} E_{k}= & -\frac{k+\alpha+2}{k+1} \frac{|a|^{2}\left(1-|a|^{2}\right)^{\alpha+2}}{1-\left(1-|a|^{2}\right)^{\alpha+2}} E_{k}(a) \\
& \times \sum_{n=0}^{\infty} \frac{n+\alpha+2}{n+1} \overline{E_{n}(a)} E_{n} \\
& +\frac{k+\alpha+2}{k+1} E_{k}, \quad \forall k \geq 0 .
\end{aligned}
$$

Proof of the Theorem 1.5 By the Eq. (5.3), we get

$$
\begin{aligned}
\left(\mathcal{B}_{\alpha, I_{a}}^{*} \mathcal{B}_{\alpha}\right)^{-1} P_{I_{a}} E_{0}= & -(\alpha+2) \frac{|a|^{2}\left(1-|a|^{2}\right)^{\alpha+2}}{1-\left(1-|a|^{2}\right)^{\alpha+2}} \\
& \times \sum_{n=0}^{\infty} \frac{n+\alpha+2}{n+1} \overline{E_{n}(a)} E_{n}+(\alpha+2) .
\end{aligned}
$$

By (5.2), we have:

$$
P_{I_{a}} E_{0}=1-\left(1-|a|^{2}\right)^{\alpha+2} \sum_{n=0}^{\infty} \overline{E_{n}(a)} E_{n} .
$$

Hence 


$$
\begin{aligned}
& \left\langle\left(\mathcal{B}_{\alpha, I_{a}}^{*} \mathcal{B}_{\alpha}\right)^{-1} P_{I_{a}} E_{0}, P_{I_{a}} E_{0}\right\rangle \\
& =-(\alpha+2) \frac{|a|^{2}\left(1-|a|^{2}\right)^{\alpha+2}}{1-\left(1-|a|^{2}\right)^{\alpha+2}}(\alpha+2 \\
& \left.\quad-\frac{\left(1-|a|^{2}\right)^{\alpha+2}}{|a|^{2}}\left(\frac{1}{\left(1-|a|^{2}\right)^{\alpha+2}}-1\right)\right) \\
& +(\alpha+2)\left(1-\left(1-|a|^{2}\right)^{\alpha+2}\right) .
\end{aligned}
$$

Since:

$$
\mathcal{B}_{\alpha}^{*}\left(P_{I_{a}} E_{0}\right)=-\left(1-|a|^{2}\right)^{\alpha+2} \sum_{n=0}^{\infty} \overline{E_{n+1}(a)} \frac{\sqrt{n+1}}{\sqrt{n+\alpha+2}} E_{n},
$$

then it is easy to check that:

$$
\mathcal{B}_{\alpha, I_{a}}^{*}\left(P_{I_{a}} E_{0}\right)=P_{I_{a}} \mathcal{B}_{\alpha}^{*}\left(P_{I_{a}} E_{0}\right)=0
$$

Thus, we get:

$$
\begin{aligned}
& \left\langle\mathcal{B}_{\alpha} \mathcal{B}_{\alpha, I_{a}}^{*}\left(P_{I_{a}} E_{0}\right),\left(P_{I_{a}} E_{0}\right)\right\rangle+\left\langle\left(\mathcal{B}_{\alpha, I_{a}}^{*} \mathcal{B}_{\alpha}\right)^{-1}\left(P_{I_{a}} E_{0}\right),\left(P_{I_{a}} E_{0}\right)\right\rangle \\
& \quad=\left\langle\left(\mathcal{B}_{\alpha, I_{a}}^{*} \mathcal{B}_{\alpha}\right)^{-1}\left(P_{I_{a}} E_{0}\right),\left(P_{I_{a}} E_{0}\right)\right\rangle \\
& \quad>2\left\|P_{I_{a}} E_{0}\right\|^{2}=2\left(1-\left(1-|a|^{2}\right)^{\alpha+2}\right),
\end{aligned}
$$

that is equivalent to:

$$
\begin{gathered}
-(\alpha+2) \frac{|a|^{2}\left(1-|a|^{2}\right)^{\alpha+2}}{1-\left(1-|a|^{2}\right)^{\alpha+2}}\left(\alpha+2-\frac{1}{|a|^{2}}\left(1-\left(1-|a|^{2}\right)^{\alpha+2}\right)\right) \\
+(\alpha+2)\left(1-\left(1-|a|^{2}\right)^{\alpha+2}\right)>2\left(1-\left(1-|a|^{2}\right)^{\alpha+2}\right) .
\end{gathered}
$$

Note that if $|a|=1$ the inequality (5.10) becomes $\alpha+2>2$, so:

$$
\begin{aligned}
& \left\langle\mathcal{B}_{\alpha} \mathcal{B}_{\alpha, I_{a}}^{*}\left(P_{I_{a}} E_{0}\right),\left(P_{I_{a}} E_{0}\right)\right\rangle+\left\langle\left(\mathcal{B}_{\alpha, I_{a}}^{*} \mathcal{B}_{\alpha}\right)^{-1}\left(P_{I_{a}} E_{0}\right),\left(P_{I_{a}} E_{0}\right)\right\rangle \\
& \quad>2\left\|P_{I_{a}} E_{0}\right\|^{2},
\end{aligned}
$$

as $|a| \rightarrow 1^{-}$.

Acknowledgements The authors are very grateful to professor Xianmin Xu for suggesting this line of research, and we also thank the referees for their excellent suggestions. This work was completed with the support of National Natural Science Foundation of China (Grant Nos. 11971087, 11871342 and 11501249) 
Open Access This article is licensed under a Creative Commons Attribution 4.0 International License, which permits use, sharing, adaptation, distribution and reproduction in any medium or format, as long as you give appropriate credit to the original author(s) and the source, provide a link to the Creative Commons licence, and indicate if changes were made. The images or other third party material in this article are included in the article's Creative Commons licence, unless indicated otherwise in a credit line to the material. If material is not included in the article's Creative Commons licence and your intended use is not permitted by statutory regulation or exceeds the permitted use, you will need to obtain permission directly from the copyright holder. To view a copy of this licence, visit http://creativecommons.org/licen ses/by/4.0/.

\section{References}

1. Aleman, A., Richter, S., Sundberg, C.: Beurling's theorem for the Bergman space. Acta Math. 177, 275-310 (1996)

2. Beurling, A.: On two problems concerning linear transformations in Hilbert space. Acta Math. 81, 239-255 (1949)

3. Hedenmalm, H., Perdomo G, Y.: Mean value surfaces with prescribed curvature form. J. Math. Pures Appl. 83, 1075-1107 (2004)

4. Hedenmalm, H., Zhu, K.: On the failure of optimal factorization for certain weighted Bergman spaces. Complex Var. Theory Appl. 19, 165-176 (1992)

5. Izuchi, K. J., Izuchi, K. H., Izuchi, Y.: Wandering subspaces and the Beurling type theorem. II. New York J. Math. 16, 489-505 (2010)

6. McCullough, S., Richter, S.: Bergman-type reproducing kernels, contractive divisors, and dilations. J. Funct. Anal. 190, 447-480 (2002)

7. Olofsson, A.: Wandering subspace theorems. Integr. Equ. Oper. Theory 51, 395-409 (2005)

8. Richter, S.: On invariant subspaces of multiplication operators on Banach spaces of analytic functions. PhD Dissertation. University of Michigan, Michigan (1986)

9. Shimorin, S.: Wold-type decompositions and wandering subspaces for operators close to isometries. J. Reine Angew. Math. 531, 147-189 (2001)

10. Shimorin, S.: On Beurling-type theorems in weighted $l^{2}$ and Bergman spaces. Proc. Am. Math. Soc. 131, 1777-1787 (2003)

11. Sun, S., Zheng, D.: Beurling type theorem on the Bergman space via the Hardy space of the bidisk. Sci. China Ser. A Math. 52, 2517-2529 (2009)

12. $\mathrm{Wu}, \mathrm{C} ., \mathrm{Yu}, \mathrm{T} . \mathrm{W}$ Wandering subspace property of the shift operator on a class of invariant subspaces of the weighted Bergman space $L_{a}^{2}\left(d A_{2}\right)$. Banach J. Math. Anal. 14, 784-820 (2020) 